\title{
L-mode-Edge Negative Triangularity Tokamak (NTT) Reactor
}

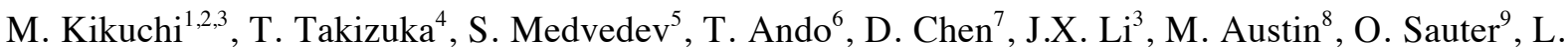

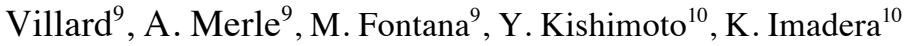

${ }^{1}$ National Institutes for Quantum and Radiological Science and Technology, Japan

${ }^{2}$ Institute of Laser Engineering, Osaka University, Japan

${ }^{3}$ Southwestern Institute of Physics, China

${ }^{4}$ Graduate School of Engineering, Osaka University, Japan

${ }^{5}$ Keldysh Institute of Applied Mathematics, RAS, Russia

${ }^{6}$ Ex-JAEA

${ }^{7}$ Key Laboratory of Neutronics and Radiation Safety, Institute of Nuclear Energy Safety Technology, Chinese Academy of Sciences, Hefei, Anhui, 230031, China

${ }^{8}$ Institute for Fusion Study, University of Texas, US

${ }^{9}$ Ecole Polytechnique Fédérale de Lausanne (EPFL), Swiss Plasma Center (SPC), CH-1015 Lausanne, Switzerland

${ }^{10}$ Institute of Advanced Energy, Kyoto University, Japan

Email: kikuchi.mitsuru@qsst.go.jp

\begin{abstract}
The negative triangularity tokamak (NTT) is a unique reactor concept based on "powerhandling-first" philosophy with the heat exhaust problem as the leading concern. The present paper exposes a reactor concept using L-mode edge based on negative triangularity tokamak (NTT) configuration, providing merits of no (or very weak) ELMs, larger particle flux and large major radius for power handling. It is shown that a reasonably compact $\left(R_{p}\right.$ from $9 m$ to $\left.7 m\right)$ NTT reactor is possible by achieving higher confinement improvement $\left(H_{H}=1.5\right)$ and/or by utilizing reasonably higher magnetic field $\left(B_{\max }=15.5 T\right)$. Current physics basis and critical issues on its scientific and technical feasibility are discussed.
\end{abstract}

\section{PHYSICS BACKGROUND OF NTT REACTOR}

\subsection{Power Handling Issues in H-mode}

The tokamak is a promising confinement configuration having possible steady-state operation [1]. The current standard reactor confinement concept relies on D-shaped plasma with H-mode confinement having the edge transport barrier. However, the H-mode edge transport barrier produces critical power handling issues in transient ELM and in steady state SOL power e-folding length. The type-I ELM produces large amount of transient energy release to the divertor plates if the edge plasma 
is in collisionless regime $\left(v^{*}<0.1\right)$ associated with the excitation of the edge bootstrap current, for example $\Delta W_{E L M} \sim 21 M J$ in ITER [2]. Such large energy loss will damage the divertor plates: minimization of ELM energy loss is necessary for ITER and the complete elimination of ELM is required for the commercial use of fusion energy. The H-mode edge transport barrier also reduces particle flux across the separatrix to the neoclassical level; balancing with the parallel semi-sonic flow gives rise to the narrow heat flux width $\lambda_{q} \sim 1 / B_{p}$ [3], [4]. Large reduction of the particle flux across the separatrix in the H-mode operation increases energy per particle to the divertor, and the formation of cold and dense, or the detached-divertor plasma becomes more challenging. For example, neoclassical particle outflux across the separatrix to a neoclassical level is an order of magnitude smaller than assumed value of fusion reactor to obtain cold and dense divertor condition [5]. The SOL density in the reactor should be high enough to form cold \& dense divertor plasma. In high-density regime $\left(n_{e}>0.85 n_{G}\right)$, degradation of H-mode confinement has been observed due to the reduced temperature gradient. All these problems originate from the H-mode system and cast a question of the feasibility of H-mode as the reactor operation mode [6], [7].

\subsection{Power Handling First}

Historically, the priority of fusion research has been "plasma confinement first" and the discovery of H-mode naturally led it to become the standard operation mode. A survey of power handling challenges and an assessment of the negative triangularity-shaped tokamak (configuration, SOL flow, confinement, MHD stability and technical merits) as an alternative operation scenario of tokamak fusion reactor was made [5]. Negative triangularity tokamak "NTT" is a reactor concept based on "power-handling-first" philosophy by locating long-leg divertor at outboard side with negative triangularity $\delta<0$ and making flux tube expansion to maximize heat exhaust surfaces. NTT configuration features a magnetic hill (i.e. specific volume $U=d V / d \Phi$ decreases with minor radius. Here $V$ and $\Phi$ are volume and toroidal flux inside a flux surface, respectively. Simply speaking, the average toroidal field over the flux surface decreases with minor radius) and NTT has never been considered as a possible fusion reactor configuration during the long history of fusion research. Locating the X-point to the large major radius side $\left(\mathrm{R}_{\mathrm{x}}>\mathrm{R}_{\mathrm{p}}\right)$, the wetted area of the divertor plates can 
be larger leading to a geometrical reduction of the heat flux. If we use double-null (DN) divertor, this leads to up-down symmetric negative triangularity tokamak, DN-NTT [8], [9]. A single-Null (SN) NTT reactor was also designed and presented at the previous IAEA-FEC conference [10].

Locating the divertor at a larger major radius position maximizes the pumping conductance for an efficient particle exhaust especially from the inboard divertor [5]. In the standard D-shaped tokamak reactor design such as SSTR [11], the pumping conductance from inboard divertor side is an order of magnitude lower than that for outer divertor side. Since experiments showed large fuel deposition near the inboard divertor plate [12], strong pumping from the inboard divertor region is quite important. We can expect comparable exhaust conductance for both divertor pumping ducts for NTT reactor since both divertor legs locate at outboard side.

\subsection{Plasma Confinement of NTT}

Shaping the plasma to negative triangularity has favorable effect on energy confinement. The trapped electron mode (TEM) is one of important candidates of the turbulent transport in tokamaks as reviewed by Kadomtsev [13]. Experiments in ASDEX [14] and Tore-Supra [15] showed that the TEM plays an important role in turbulent transport in collisionless tokamaks. The TEM growth is enhanced by the precession drift resonance since the perturbed distribution function $g_{e} \sim 1 /\left(\omega-\omega_{D e}\right)$, where $\omega_{D e}$ is the trapped electron toroidal precession drift frequency. Drift de-resonance of the TEM is possible through number of ways, such as finite beta by Rosenbluth [16], Shafranov shift at high $\beta_{\mathrm{p}}$ operation by Connor [17], negative shear by Kadomtsev [18], elongation and negative triangularity by Rewoldt [19]. Among these mechanisms, the combination of negative shear, high $\beta_{p}$ operation and the negative triangularity leads to an attractive operation scenario for the steady-state tokamak reactor. A comparative study of positive and negative triangularity tokamak plasmas in TCV during electron cyclotron (EC) heating [20], [21] (Figure 1) clearly demonstrated NTT can double the electron energy confinement time in the TEM-dominated regime without H-mode. TCV also compared the electron temperature fluctuation between NTT and standard D-shaped plasma (positive triangularity tokamak, PTT). Fontana [22] measured the turbulent electron temperature fluctuation $\delta T_{e} / T_{e}$ a using CECE (correlation electron cyclotron emission) in ohmically-heated low density plasmas. TCV's ohmically- 
heated NTTs have better confinement than PTTs. They showed a clear reduction of both fluctuation amplitude and correlation length in NTT at normalized radius range $0.5<\rho_{\mathrm{vol}}<0.8$ where $\mathrm{T}_{\mathrm{e}}>\mathrm{T}_{\mathrm{i}}$. GENE simulation for these discharges implies dominant mode is TEM [23] similar to the case of EC heating results in [21].

Another explanation of NTT improved confinement from the non-stiffness of the L-mode edge was made by Sauter [24]. He found that the core region $(r / a<0.8)$ is stiff for heat transport above the critical temperature gradient but the edge region $(0.8<r / a<1.0)$ is not stiff, suggesting different transport mechanisms dominate. He observed experimentally that L-mode edge in NTT has reduced transport, that may explain the TCV NTT improved confinement results [21]. Merlo [25] showed that the critical temperature gradient increases toward the edge and further increases in the negative triangularity consistent with Sauter's observation. Furthermore, Fontana's measurements of turbulent temperature fluctuation $\delta T_{e} / T_{e}$ as a function of $R / L_{T e}$ in TCV [22] showed that the critical temperature gradient $\left(R / L_{T e}\right)_{\text {crit }}$ in NTT is larger than that in PTT consistent with Merlo's predictions. Resistive ballooning mode (RBM) is a possible candidate of edge and SOL turbulent transport. The RBM is localized along the field line in the bad curvature region. Riva [26] analyzed the linear and nonlinear RBMs showing that the RBMs in the vertically elongated DN-NTT are more stable. The physical explanation of the stabilizing mechanism is that the magnetic field line pitch is large near the outer mid-plane and the particle residence time in bad curvature region is shorter. This mechanism may be commonly applicable for all types of ballooning-like instabilities in DN-NTT.

Recently, DIII-D [27], [28], [29] made a groundbreaking experiment demonstrating $H_{H}=1.2$ and $\beta_{N}=2.6$ simultaneously with L-mode-like edge in NTT showing that the proposed NTT reactor condition $\left(H_{H}=1.12, \beta_{N}=2.1\right)$ [10] can be exceeded. DIII-D has flexible shaping capability to produce up-down symmetric inner-wall limited near-DN NTT configuration (see Figure 1). While inner-wall limited auxiliary heated D-shaped plasma went to the limiter H-mode $\left(\mathrm{Z}_{\text {eff }}(0)\right.$ went up to 3.5-4 after L-H transition from 2.0-2.5 in L-mode phase), NTT stayed at L-mode $\left(\mathrm{Z}_{\text {eff }}(0)\right.$ stays at 2.0-2.5) with improved confinement to achieve $H_{H}=1.2$ at $\beta_{N}=2.6$. Impurity contamination is lower and the neutron emission was doubled in NTT as reported by Austin [29]. These DIII-D NTT plasmas are relatively 
high density $\left(\mathrm{n}_{\mathrm{e}}(0) \sim 6 \times 10^{19} \mathrm{~m}^{-3}\right)$ but still in the mixed ITG/TEM regime dominated by TEM as shown by Marinoni [30].

\subsection{L-mode Edge Operation}

Current tokamak reactor designs are based on the H-mode operation. Considering the powerhandling issues given above, the L-mode edge is preferred as a robust operation mode in fusion reactor. L-mode also provides much larger particle flux than H-mode necessary to form cold \& dense divertor (i.e. neoclassical particle flux in H-mode is too small [5]). DIII-D experiments [29] showed that inner-wall limited NTT edge stayed in L-mode even at large NB heating power $\left(P_{N B}=11 M W\right)$, while inner-wall-limited D-shaped plasma went to the limiter $\mathrm{H}$-mode for a same heating power. The SOL density in DIII-D NTT L-mode edge is much higher than that of H-mode. Since the key impurity control mechanism in the fusion reactor is the parallel friction force to compete with the thermal force to prevent impurity back flow, sustainment of high SOL density is key to the impurity control.

While L-mode edge turbulent transport may be weaker in NTT than that of L-mode edge PTT [26], it will provide a better solution for the reactor power and particle exhaust than those by the Hmode operation of PTT. Research toward the sustainment of L-mode edge with improved confinement at reactor-relevant normalized beta is desired in large higher field negative triangularity tokamaks as well as the establishment of L-H power threshold scaling on negative triangularity.

While all these experimental and theoretical results are encouraging for the proposed NTT reactor with L-mode edge, TCV and DIII-D experiments are of inner-wall limited plasmas. Respective roles of inner-wall limiter and X-point operation are important missing pieces of information. For the latter subject, it reminds us that the original JT-60 had divertor X-point in the outboard mid-plane and showed very short period of H-mode but returned to L-mode confinement [31]. Possible sustainment of L-mode edge in a reactor is further discussed in section 3.2.

\subsection{MHD Stability of NTT}

The MHD stability of NTT has been studied in our previous papers by Medvedev [9], [10]. Since NTT magnetic configuration features magnetic hill, the localized Mercier mode stabilization is only possible with finite magnetic shear, i.e. $1 / 4>D_{M}$, where $1 / 4$ comes from shear stabilization of the localized mode and $D_{M}$ is the Mercier index, where $D_{M}>0$ corresponds to the magnetic hill 
$\left(D_{M}=8 \pi r(d p / d r)\left(1-q^{2}\right) / s^{2} B^{2}\right.$ in circular plasma). Ohkawa's comet shape $(\kappa<1$ and $\delta<0)$ [32] is the exception of negative triangularity with magnetic well, where a single X-point is located in the inboard side. Mercier stability is significantly modified including the kinetic effects such as thermal trapped ion effect given by Kruskal-Oberman [33] and fast ion effect, especially in the central region, where the stability condition is given by $1 / 4>D_{M M}=D_{M}+D_{K O}+D_{h}, D_{K O} \sim-0.47\left(8 \pi p_{i} / s^{2} B^{2}\right) /(r / R)^{0.5}$ is the Kruskal-Oberman correction and $D_{h} \sim q^{2}(2 R r)^{0.5} d p_{h} / d r / s^{2} B^{2}$ is the energetic ion correction as discussed by Porcelli [34].

Medvedev [9] studied and optimized the ideal MHD stability of a $\kappa_{x}=1.5$ DN-NTT. The DNNTT is characterized by the high magnetic shear near the separatrix. Stable high-normalized beta equilibrium is obtained by setting $d p / d \psi$ close to Mercier stability limit in the inner half region $\left(/ \psi / /^{1 / 2}<0.5\right)$ as well at the very edge considering the high edge magnetic shear. To take the kinetic stabilization mentioned above into account, the case of higher pressure-gradient in the inner half region could be considered. Ballooning mode sets $d p / d \psi$ profile in the outer half region $\left(\left(/ \psi / /^{1 / 2}>0.5\right)\right)$. Using this basic idea, stability limit to ideal MHD modes was studied. The beta limit of NTT strongly depends on $l_{i}$ (internal inductance). For $A=2.6\left(R_{p}=7 m, a_{p}=2.7 m, \kappa=1.5, \delta=-0.9\right), I_{N}=I_{p} / a_{p} B_{t}=0.9$ and $l_{i}=0.9$ (positive shear; PS), the beta limit is $\beta_{N}=3.2$ without wall stabilization and $\beta_{N}=3.3$ with wall stabilization $\left(a_{w} / a_{p}=1.3\right)$ [9]. If we reduce $l_{i}=0.75$, the beta limit is reduced to $\beta_{N}=2.4$ without wall stabilization and $\beta_{N}=2.6$ with wall stabilization. While ballooning mode is stable for negative shear (NS) region, NS configuration has relatively low beta limit $\beta_{N}=2.1$ without wall stabilization. This is marked difference with PTT NS plasma. The DN-NTT is more prone to the axisymmetric instability. Relatively close fitting $6 \mathrm{~cm}$ steel stabilizing shell at $a_{w} / a_{p}=1.25$ is needed to reduce the growth rate of $n=0$ axisymmetric mode $\gamma_{n=0}=95 s^{-1}$, which requires good feedback control of axisymmetric mode [9].

More elongated NTT can be stable to the axisymmetric modes $(n=0)$ for the single-null divertor (SN-NTT). Medvedev studied the stability of SN-NTT for $A=3\left(R_{p}=9 m, a_{p}=3 m, \kappa=1.8, \delta_{u}=-0.4\right.$ and 0.07, $\left.\delta_{l}=-0.9\right), I_{N}=1.19\left(I_{p}=21 M A, B_{t}=5.86 T\right)[10]$. For an upper triangularity $\delta_{u}=-0.4$ and $l_{i}=0.84$, the beta limit is $\beta_{N}=2.79$ without wall stabilization and $\beta_{N}=3.3$ with wall stabilization $\left(a_{w} / a_{p}=1.3\right)$ while edge magnetic shear is smaller than that of DN-NTT. For this configuration, the growth rate of the 
axisymmetric mode with $a_{w} / a_{p}=1.3$ is $\gamma_{n=0}=14 s^{-1}$ for $6 \mathrm{~cm}$ steel stabilizing shell. For an upper triangularity $\delta_{u}=-0.07$ and $l_{i}=0.84$, the beta limit is $\beta_{N}=3.14$ without wall stabilization and $\beta_{N}=3.56$ with wall stabilization $\left(a_{w} / a_{p}=1.3\right)$. The growth rate of the axisymmetric mode with $a_{w} / a_{p}=1.3$ is $\gamma_{n=0}=11 \mathrm{~s}^{-1}$ for $6 \mathrm{~cm}$ steel stabilizing shell for this configuration.

We studied the contribution of edge pedestal in optimized pressure profile in NTT. Figure 2 a) shows optimized pressure profile in NTT. Pressure should have near-flat profile in the central region. Figure $2 \mathrm{~b}$ ) shows the radial profile of $p^{\prime}=d p / d \psi$ for a typical $\mathrm{A}=3, \kappa=1.8$ SN-NTT equilibrium optimized for ideal MHD stability. The inner half is shaped to have Mercier-limiting profile, the outer half is ballooning-limiting profile and the very edge is designed to have Mercier-limiting profile. To demonstrate non-negligible pedestal contribution to the beta limit, we set $d p / d \psi$ smoothly go to zero in the pedestal region. We found 5\% reduction in normalized beta.

Reduction of the pedestal beta limit is one of the important targets of the NTT reactor design to minimize energy loss during the ELM event. Merle [35] made EPED-CH calculations and showed that the pedestal pressure for the NTT is 4 times less than PTT even if it goes to H-mode, a similar reduction can be expected for ELM energy losses since these two quantities are often correlated. Merle identified that the key parameter is the average triangularity $\delta=\left(\delta_{l}+\delta_{u}\right) / 2$. Figure 3 shows the normalized pedestal pressure calculated by EPED-CH as a function of the average triangularity $\delta$. Typically $P_{p e d}=9.2 \mathrm{kPa}(0.1 \%$ of magnetic pressure $)$ will be a limiting pedestal pressure in NTT reactor much smaller than the typical positive triangularity tokamak value of $P_{p e d}=39 \mathrm{kPa}(0.4 \%$ of magnetic pressure). Thus, the edge bootstrap current to excite surface kink modes is expected to be small. While NTT has lower pedestal pressure and lower ELM energy loss if it happens, reactor operation will need complete suppression of ELM [36]. Enhanced pedestal radiation and SOL radiation to sustain L-mode edge without ELM will be a key. Also a detached or semi-detached divertor has to be carefully controlled. Lower pedestal pressure limit will certainly help to minimize the damage of plasma facing components in case of off-normal events. 


\section{TECHNICAL DESIGN OF NTT REACTOR}

\subsection{Configuration Designs}

The NTT configuration design was started using the inverted D-shaped TFC for the DN-NTT [9]. Figure 4 shows revised PFC designs for DN-NTT with reduced elongation $\left(\kappa_{\mathrm{x}}=1.5\right)$ to meet the vertical stability of DN-NTT. This configuration can be realized by a reasonable set of equilibrium coils $\Sigma I_{P F} / I_{p}=6.7$ due to closer equilibrium coils. However, mechanical support for the centering and bending forces of TF coils are expected to be quite challenging.

Considering the above difficulty, we decided to use racetrack shape TF coils, where we can adopt standard wedge support for the centering force. We also decided to move all internal PC coils outside of the TFC for easier manufacturing. We adopted radial disk structure used in ITER and SSTR [11] to ensure radial force transmission. With all these changes, equilibrium shaping-coils are more distant from the NT-shaped plasma. Considering the better positional stability, reasonable normalized beta limit and the up-down power sharing issue in DN, we adopted the SN configuration for the NTT with modest confinement improvement factor $H_{H}=1.12$. The maximum field is limited to $B_{\max }=13.6 \mathrm{~T}$ due to stress limit $800 M P$ and the aspect ratio $A=R_{p} / a_{p}=3$. This gives rise to a large reactor size and current, i.e. $\mathrm{I}_{p}=21 M A, R_{p}=9 m, B_{t}=5.86 T, E_{T F C}=170 G J$ with the TF coil dimension of $15 \mathrm{~m}$ width and $20 \mathrm{~m}$ height. Major coil parameters of Figure 5 a) are shown in Table 1 . Number of TF coils is 18 . Total Ampere Turns is $263 M A T$ with a conductor current of $126 \mathrm{kA}$. Number of turns/coil is 116 and the winding structure is double pancake using 6 radial disks. The discharge constant of TF coil is 12 second corresponding to the terminal voltage of $12.5 \mathrm{kV}$ for quench protection assuming quench detection is within 2 second. TF conductor is designed using the standard ITER-like Nb3Sn superconductors design criteria as given in the next subsection. The set of poloidal field (PF) coils enables the shaping of upper plasma in order to vary its triangularity, $\delta_{u}=-0.4$ (orange) to $\delta_{u}=-0.07$ (blue), with vertical elongation $\kappa_{x}=1.8$ at the separatrix and $q_{95}=3.0$ and $q_{95}=3.1$, respectively. We obtain these shaping variations with $\Sigma I_{P F} / I_{p}=6.8$ and $\Sigma I_{P F} / I_{p}=5.9$, respectively. These values are comparable to that of DN-NTT with inverted-shaped TF coil. 
To construct a more compact fusion reactor, we made a parameter survey through the major radius $\left(R_{p}=9-7 m\right)$ scan for $A=3$ and $A=3.5$ (Figure 6) while keeping the confinement measure, $H_{H} I_{p} A=69.3 \mathrm{MA}$, and the engineering safety factor, $q_{\text {eng }}=3.5$. We also kept the normalized density $n / n_{G W}=0.85$. Procedures in this parameter scans are introduced in the appendix A.

We increased $\kappa_{x}=1.8$ to $\kappa_{x}=2.0$ for the $A=3$ scan while $n=0$ growth rate becomes $\gamma_{n=0}=40 \mathrm{~s}^{-1}$. This could be managed by the active feedback control. We assumed $\kappa_{x}=1.85$ for the $A=3.5$ scan. In the parameter scan, $H_{H}$ is fixed in solid line $H_{H}=1.2$ and $H_{H}$ is increased from 1.1 to 1.5 as $R_{p}$ is reduced from $9 m$ to $7 m$ in broken line.

Parameter surveys for the $A=3$ case are shown in Figure 6 a) and b). Solid lines of Figure 6 a) and b) show that reduction of major radius to $R_{p}=7 \mathrm{~m}$ is possible while plasma current $I_{p}$ and $\beta_{N}$ are almost kept constant but it requires higher magnetic field $B_{t} \sim 16 T$ to keep $q_{\text {eng }}=3.5$. Moreover, the fusion power $P_{f}$ and the average neutron flux $q_{n}$ also increase. Broken lines of Figure 6 a) and b) show that reduction of major radius to $R_{p}=7 \mathrm{~m}$ is also possible with improved confinement $\left(H_{H}=1.5\right)$ while $B_{\max }$ is nearly constant. In this case, the fusion power is reduced to $P_{f}=2 G W$ and the neutron wall load stays almost constant $q_{n} \sim 1.4-1.5 \mathrm{MW} / \mathrm{m}^{2}$ while the normalized beta $\beta_{N}$ becomes higher than the case for $R_{p}=9 m\left(\beta_{N}=2.1\right)$. Based on this parameter scan, we finalized the parameter set of the steady-state operation point using the system code shown in subsection 2.3 as $I_{p}=15.4 M A, B_{t}=5.94 T, H_{H}=1.5$, $n=0.77 \times 10^{20} \mathrm{~m}^{-3}, P_{f}=2.07 \mathrm{GW}, B_{\max }=13.5 T, \beta_{N}=2.7, P_{C D}=106 \mathrm{MW}, Q=19.5$. Configuration design for highly shaped NTT with upper triangularity $\delta_{u}=-0.4$ and lower triangularity $\delta_{l}=-0.9$ is realized by a set of shaping coils with $\Sigma I_{P F} / I_{p}=8.1$. The coil configuration is shown in Figure $5 \mathrm{~b}$ ). Since $B_{\max }=13.5 \mathrm{~T}$, standard wedge support allows maximum stress within $800 \mathrm{MPa}$ with the TF coil dimension of $12 \mathrm{~m}$ width and $16 \mathrm{~m}$ height. TF magnetic energy is reduced to $86.5 G J$ with the total Ampere Turns of 207MAT with the conductor current $100 \mathrm{kA}$. Number of turns/coil is 116 and the winding structure is double pancake using 6 radial disks. The discharge constant and the terminal voltage are 10 second and $10 \mathrm{kV}$, respectively.

Parameter surveys for the $A=3.5$ case $\left(\kappa_{x}=1.85\right)$ are shown in Figure $\left.6 \mathrm{c}\right)$ and d). Solid lines of Figure $6 \mathrm{c}$ ) and d) show that reduction of major radius to $R_{p}=7 \mathrm{~m}$ is also possible while plasma current 
$I_{p}$ and $\beta_{N}$ are almost kept constant but it requires higher magnetic field $B_{t} \sim 19.5 T$ for fixed $H_{H}=1.2$ to keep $q_{\text {eng }}=3.5$. The plasma volume is smaller $\left(V_{p} \sim 1000 m^{3}\right)$ compared with the $A=3$ case $\left(V_{p} \sim 1500 m^{3}\right)$. Broken lines of Figure $5 \mathrm{c}$ ) and d) are parameter survey for $A=3.5$ case with varying $H_{H}$ factor and show that reduction of major radius to $R_{p}=7 \mathrm{~m}$ is also possible with improved confinement $\left(H_{H}=1.5\right)$. Final parameter set of the steady-state operation is determined by the system code as, $I_{p}=13.2 \mathrm{MA}$, $B_{t}=7.53 T, n=0.9 \times 10^{20} \mathrm{~m}^{-3}, P_{f}=1.9 \mathrm{GW}, B_{\max }=15.5 T, \beta_{N}=2.5, P_{C D}=115 \mathrm{MW}, Q=21.2$. Similar shaping with upper triangularity $\delta_{u}=-0.4$ and lower triangularity $\delta_{l}=-0.9$ is realized by a set of shaping coils with $\Sigma I_{P F} / I_{p}=8.25$. The coil configuration is shown in Figure $5 \mathrm{c}$ ). Since $B_{\max }=15.5 T$, wedge support region is extended toward the machine center to keep maximum stress below $800 \mathrm{MPa}$. The TF coil dimension is $11 \mathrm{~m}$ width and $14 \mathrm{~m}$ height. TF magnetic energy is $104 G J$ with total Ampere turn of $263 M A T$ with the conductor current $100 \mathrm{kA}$. Number of turns/coil is 146 and the winding structure is double pancake using 7 radial disks. The discharge constant and the terminal voltage are 12 second and $10 \mathrm{kV}$, respectively. TF conductor is designed using the standard ITER-like $N_{b 3} S_{n}$ superconductors design criteria and also more advanced Bi2212 high $T_{c}$ superconductor as given in the subsection 2.2.

For all three TF magnet designs, we selected discharge constant to keep the quench temperature rise of $150 \mathrm{~K}$.

\subsection{Conductor design of TF coils}

For the three reactor configurations above, we developed two conductor designs for each TF coil. We assumed high $J_{c}\left(1000 \mathrm{~A} / \mathrm{mm}^{2}\right) \mathrm{Nb3Sn}$ strand for the design extrapolated from recent development of high $J_{c}$ strand for ITER [37]. Design parameters of these six conductors are shown in Table 2 and their conductor shapes are shown in Figure 7. Our design utilized $J_{c}(B, T, \varepsilon)$ parameterzation by Bottura [38] for Nb3Sn and the paper from Rey [39] for Bi2212.

In the $A=3, R_{p}=9 m$ designs with $B_{\max }=13.6 T$ (Figure 5 a)), we can use $N b 3 S n$ cable-in-conduit (CIC) conductor with $S S$ conduit or $T_{i}$ conduit at $5.0 \mathrm{~K}$ operating temperature. The TF conductor of ITER uses $S S$ conduit but the thermal expansion coefficient of SS is different from that of $N b 3 S n$ strand and has to assume a large strain. The $N b 3 S n C I C$ conductor with $S S$ conduit is designed to 
allow higher total operating strain $(-0.6 \%)$. The ratio of $\mathrm{SC}$ and $\mathrm{Cu}$ strands is $2: 1$ with a void fraction of $33.3 \%$.

Titanium $\left(T_{i}\right)$ has a thermal expansion coefficient close to $N b 3 S n$ superconductor and is effective to reduce strain of the SC. Ando [40] proposed to use $T_{i}$ as a jacket of superconductor and $T_{i}$ -jacket conductor has been manufactured as the ITER TF insert conductor [41] and the effectiveness of $T_{i}$ jacket is demonstrated [42]. The design strain can be reduced from $-0.6 \%$ in SS jacket to $-0.05 \%$ in $T_{i}$ jacket. Reduction of design strain leads to a significant increase in operating critical current density. Therefore, we can increase $\mathrm{Cu} /$ non- $\mathrm{Cu}$ ratio from 1.0 to 2.0 (or reduce amount of $\mathrm{Nb3Sn}$ in the strand). Ratio of $\mathrm{SC}$ and $\mathrm{Cu}$ strands is also changed from $2: 1$ to $5: 4$ as seen Figure 7 . The cable diameter is reduced from $66 \mathrm{~mm}$ to $61.8 \mathrm{~mm}$. The conductor has central cooling channel with the outer diameter $O D=11 \mathrm{~mm}$ and the inner diameter $I D=9 \mathrm{~mm}$ for the supercritical Helium forced flow cooling.

In the $A=3, R_{p}=7 m$ configuration, conductor design is similarly made for $S S$ conduit and $T_{i}$ conduit. For a $S S$ conduit design, total operating strain is $-0.6 \%$ and the $\mathrm{Cu} / \mathrm{Non} \mathrm{Cu}$ ratio is 1.0 and the ratio of $\mathrm{SC}$ and $\mathrm{Cu}$ strands is $2: 1$ with a void fraction of $33.3 \%$. For a $T_{i}$ conduit design, total operating strain is $-0.05 \%$ and the $\mathrm{Cu} / \mathrm{Non} \mathrm{Cu}$ ratio is 2.0 and the ratio of $\mathrm{SC}$ and $\mathrm{Cu}$ strands is $1: 2$ with a void fraction of $33.3 \%$. Helium coolant channel design is same for $A=3, R_{p}=9 m$ configuration.

In the $A=3.5, R_{p}=7 m$ configuration with $B_{\max }=15.5 \mathrm{~T}$, we can also use $N b 3 S n$ cable-in-conduit (CIC) conductor at $5.0 \mathrm{~K}$ operating temperature if we use $T_{i}$ conduit. The $S S$ conduit adopted for ITER will have difficulty by a large reduction of $J_{c}$ due to the large strain due to the difference in thermal expansion coefficient between $\mathrm{SS}$ and $\mathrm{Nb} 3 \mathrm{Sn}$. High $T_{c}$ superconductor is attractive technology for the magnetic confinement fusion providing compact reactor configuration such as VECTOR [43] where the high $T_{c}$ conductor designs are successfully developed [44], [45]. As long as $\mathrm{Nb3Sn}$ design is possible, it is more reliable and economical at present to use $\mathrm{Nb} 3 \mathrm{Sn}$ design. However, recent development of high $T_{c}$ superconductor is quite remarkable and the critical current density of Bi2212 at $15 T$ exceeded that of $\mathrm{Nb3Sn}$ as reported by J. Jiang [46]. This makes it reasonable to give a design option using the Bi2212 at an operating temperature of $20 \mathrm{~K}$ under moderately high magnetic field (15.5T). At this elevated temperature, the heat capacity of $P b S n$ alloy is quite large and Bi2212 strands are impregnated in $\mathrm{PbSn}$ alloy and its conductor size is comparable with $\mathrm{Nb3Sn}$ as shown in Figure 7. 
The maximum allowable stress of cryogenic steel will be reduced by $7 \%$ at $20 \mathrm{~K}$ but an addition of backing cylinder between CS coil gap will satisfy the stress requirement.

\subsection{System code assessment of steady-state operation in NTT Reactor}

The current tokamak system code is based on the shaped cross section equilibrium database. However, the negative triangularity equilibrium database is not included and the code was not applicable for NTT. Sauter expanded the equilibrium database to include NTT [47] and the resultant formula is included in the system code by Chen [48]. Results of the system code for three configurations in Figure 5 are summarized in Table 3. First parameter set from left corresponding to Fig. 5 a) is the single-null negative triangularity tokamak (SN-NTT) reactor proposed in the last Fusion Energy Conference (2016) [10]. This reactor has fusion power $P_{F^{\sim}} 3 G W$ and the transport power across the separatrix is $P_{\text {trans }} \sim 594 M W$. It operates at relatively lower density $n_{e}=6.3 \times 10^{19} \mathrm{~m}^{-3}$ for the steady-state operation with the current drive power of $P_{C D}=168 \mathrm{MW}$.

Newly proposed reactor parameters are shown in $2^{\text {nd }}$ and $3^{\text {rd }}$ set of parameters in Table 3 . We found that $\mathrm{Q} \sim 20$ steady state operation may be possible even at reduced size machines if high confinement $\left(H_{H}=1.5\right)$ is achievable for $A=3$ case and also if both high field $\left(B_{\max }=15.5 T\right)$ and high confinement $\left(H_{H}=1.5\right)$ are achievable for $A=3.5$ case. Operating normalized beta in $A=3$ and $A=3.5$ are within no-wall limits. Here $\beta_{N}{ }^{N W}=2.89(A=3)$ and $\beta_{N}{ }^{N W}=2.87(A=3.5)$. Transport powers across the separatrix are $384 M W(A=3)$ and $338 M W(A=3.5)$, respectively. The power required for steadystate operation is $96.6 \mathrm{MW}$ for $A=3$ design and $101 \mathrm{MW}$ for $A=3.5$ design, respectively.

\subsection{Divertor and FTE (Flux Tube Expansion)}

Locating the divertor hit-point on the large major radius side provides the opportunity of a larger wetted area. Long-leg divertor also contributes to reduce the divertor heat flux through radiation between X-point to hit-point. However, magnetic pitch angle increases rapidly away from the X-point and the width of the flux tube becomes narrower. The "Flux-Tube-Expansion divertor" concept [49] is adopted to reduce magnetic pitch near the divertor plate. An important merit of NTT is that FTE can be applied in both inner and outer divertor legs. Herrmann [50] gave a minimum field line angle of $1^{\circ}$, which is limited by the divertor alignment in the toroidal direction. Figure 8 show typical NTT equilibria without and with FTE $\left(I_{F T E}=2.5 M A\right)$. Without FTE, the angles between the 
magnetic field line and the divertor plate surface at outer and inner divertor plates are $\alpha=8.7^{\circ}$ and $\alpha=5.1^{\circ}$, respectively. Application of FTE $\left(I_{F T E}=2.5 M A\right)$ reduces these angles to $\alpha=2.1^{\circ}$ for outer divertor plate and $\alpha=2.6^{\circ}$ for inner divertor plate, respectively. Dramatic reduction of field line angle is possible especially for the outer divertor.

Figure 9 shows the divertor geometries with FTE-coils of $I_{p}=15 M A, R_{p}=7 m$ NTT configuration. Separatrix line is shown in red (color online). A $1 \mathrm{~mm}$ SOL flux tube width at the outboard mid-plane are projected to a $3.7 \mathrm{~mm}$ width flux tube in the inboard mid-plane, a $4.3 \mathrm{~mm}$ width flux tube in the inboard plate, and $4.8 \mathrm{~mm}$ width flux tube in the outboard divertor plates (black lines). If the divertor plates are more inclined (green lines), grazing angles are close to 1 degree and the length along the plates can be $10 \mathrm{~mm}$ as shown in Figure 9. Assuming the SOL heat flux width of NTT L-mode edge is twice of that of PTT H-mode, the heat flux width at the divertor plates could be $20 \mathrm{~mm}$, each. We may expect wider heat flux width near the detached condition as well [51].

\section{DISCUSSIONS}

The scientific feasibility of operating NTT as a reactor is still much less well established than for a PTT due to lack of experimental and theoretical investigations. Here we address some key questions and provide implications to future scientific research.

\subsection{Feasibility of Improved Confinement in DEMO Reactor Regime}

Many of proposed DEMOs such as EU-DEMO $\left(R_{p}=9 m, P_{f u s}=2 G W\right)$ operated in high-density regime $\left(n_{e}>10^{20} \mathrm{~m}^{-3}\right)$ may be in the ITG dominant regime. A key question is if the proposed NTT reactors can have improved confinement with L-mode edge. The proposed steady-state operations of NTT reactor will be at lower densities $\left(n_{e}=0.6-0.9 \times 10^{20} \mathrm{~m}^{-3}\right.$ in Table 3$)$ due to a limitation of noninductive current drive efficiency. We still do not know if the reactor can have required improved confinement in these density regimes while recent experiments in TCV and DIII-D are encouraging (especially high density DIII-D operation is in the TEM-dominant regime). Further progress of experimental and theoretical efforts as well as turbulent transport modeling efforts for the NTT reactor may provide better physics basis for the NTT reactor. 
Considering the recent experimental and theoretical progress, local linear TEM stability may be not the sole reason for the actual confinement improvement observed in TCV and DIII-D. Improved confinement may be also enhanced by the better stability against the RBM in the non-stiff region $(0.8$ $<r / a<1)$ in the L-mode within the lowered pedestal pressure limit. Near the magnetic axis, both NTT and PTT have almost zero triangularity but still have improved confinement in both TCV and DIII-D. This may be a non-local effect [52], the size scaling of which is presently unknown. Further global nonlinear gyrokinetic simulations are needed in order to determine whether the effect survives in a reactor-size NTT. Also reduced temperature fluctuation in NTT relative to PTT is observed even in $T_{e} / T_{i} \sim 1$ ITG/TEM mixed regime with a collisionality range $0.3<1 / v_{\text {eff }}<1$ in recent TCV experiments [23]. DIII-D high beta results at $n_{e}(0) \sim 6.0 \times 10^{19} \mathrm{~m}^{-3}$ [30] also implies that improved confinement can exist even under the ITG/TEM mixed regime.

\subsection{Avoiding L-H Transition in Reactor Regime}

One of the key proposals in this paper is to stay in the L-mode edge in the NTT reactor. According to the ITER physics basis [53], $\mathrm{P}_{\mathrm{LH}}(\mathrm{MW})$ scales as $P_{L H}=0.042 n_{20}{ }^{0.73} B_{t}^{0.74} S^{0.98}$. Here $n_{20}, B_{t}$ and $S$ are electron density $\left(10^{20} \mathrm{~m}^{-3}\right)$, toroidal field (T), and surface area $\left(\mathrm{m}^{2}\right)$, respectively. Values of $P_{\text {heat }} / P_{L H}$ in DIII-D NTT and PTT are both $\sim 6.7$ and NTT stays in L-mode edge and PTT made L-H transition. The values of $P_{\text {transp }} / P_{L H}$ for three reactors in Table 3 are $3.5\left(A=3, R_{p}=9 m\right), 2.2(A=3$, $\left.R_{p}=7 \mathrm{~m}\right)$, and $2.6\left(A=3.5, R_{p}=7 \mathrm{~m}\right)$, respectively. Considering the DIII-D NTT stays at L-mode edge even at 6.7 times L-H transition power scaling, there might be a possible way to keep NTT reactor edge staying in L-mode edge.

Inner-wall contact may have an important role in suppressing the L-H transition but the contact area in DIII-D NTT is much smaller than PTT limiter H-mode. The absence of X-point is another critical issue left for future study, while the existence of limiter H-modes in various tokamaks JFT-2M [54], JT-60 [55], JIPP T-IIU [56], TCV [57] implies X-point is not essential for the L- to H-mode transition. The role of negative triangularity on the L- to H-mode transition is a crucially important experimental and theoretical subject for future research. If the power threshold for L- to H-mode transition in NTT in divertor configuration is lower than transport power in Table 3, enhanced core 
and edge radiation may be needed to supress L- to H-mode transition to stay at L-mode edge. Note that designing NTT SN magnetic field towards unfavorable direction should further ensure an L-mode state.

\subsection{Possible power handling scenario in NTT reactors}

Fusion reactor needs to operate stably and reliably for year-long operation. Current database for long pulse operation is still very limited even for PT tokamak as well as stellarators as shown in Figure 10 [57], [58]. Considering the heat flux level of existing power system such as fission power plants $\left(q_{\max } \sim 1 \mathrm{MW} / \mathrm{m}^{2}\right)$ and fossil power plants $\left(q_{\max } \sim 0.3 \mathrm{MW} / \mathrm{m}^{2}\right)$, the maximum heat flux of magnetic fusion power plant may need to be reduced to a few $M W / \mathrm{m}^{2}$.

For example, case for $A=3, R_{p}=9 \mathrm{~m}$ configuration, the total heating power is $P_{\text {total }}=761 \mathrm{MW}$, of which $167 \mathrm{MW}(22 \%)$ are radiated in the core-region. Further $20 \%(150 \mathrm{MW})$ could be radiated in the L-mode edge region, which could be free from confinement degradation. High SOL density in the Lmode edge would help to further radiate in the SOL region and also long-leg (X-point to divertor plate : $2.6 \mathrm{~m})$ divertor, which could radiate $\sim 50 \%$ of total heating power [58]. Remaining $8 \%(\sim 60 \mathrm{MW})$ would be handled by two divertor plates with total effective wetted area of $S_{\text {plate }}=30 \mathrm{~m}^{2}$ to handle radiation, conduction and convection heat flux to stay at a few $\mathrm{MW} / \mathrm{m}^{2}$ heat flux. Since toroidal length at the divertor hit points are $70 \mathrm{~m}$ for inboard divertor plate and $88 \mathrm{~m}$ for outboard divertor plate, effective poloidal length of the divertor power handling area (D-PHA) of $0.2 \mathrm{~m}$ for each plate would be sufficient. Precise control of power inlet to the D-PHA is a critical R\&D issue.

In the European DEMO scenario [51], higher core radiation fraction is envisaged but is limited to assure L- to H-mode transition by the condition $P_{\text {sep }}>1.3 P_{L H}$. On the other hand, the NTT reactor may offer possible high core radiation scenario to stay at L-mode edge and to reduce divertor heat load.

\section{SUMMARY}

Current power handling challenge in tokamak D-shaped H-mode is quite serious and world fusion research effort is devoted to resolve this problem with some success. However, we still do not know if we can open the way of power handling for year-long operation in this route. The Negative Triangularity Tokamak (NTT) reactor concept is proposed as an alternative route for tokamak power 
handling. NTT now has good experimental and technical basis while there are still many physics issues to be clarified in the experiment and simulation.

\section{ACKNOWLEDGEMENT}

The authors appreciate TCV experimental and DIII-D experimental teams as well as their theory and simulation teams to clarify key physics issues related to NTT configuration. The authors also appreciate valuable constructive comments by the referees. This work was supported in part by the Swiss Naitonal Science foundation and carried out in part within the framework of the EUROfusion Consortium and has received funding from the Euratom research and training programme $2014-2018$ and $2019-2020$ under grant agreement No 633053. The views and opinions expressed herein do not necessarily reflect those of the European Commission.

\section{APPENDIX A}

We briefly describe equations used for the parameter scan in subsection 2.1 . While there is some possibility that NTT confinement scales differently, we kept $H_{H} I_{p} A$ constant during the parameter scan $\left(H_{H} I_{p} A=69.3 \mathrm{MA}\right.$ in the present study) since it is a good measure of fusion performance as discussed in ITER physics basis [59]. Here $H_{H}$ is confinement enhancement factor over the IPB98(y,2) scaling [60], $I_{p}$ is plasma current and $A=R_{p} / a_{p}$ is aspect ratio.

A relation between $I_{p}$ and $B_{t}$ (toroidal magnetic field at the plasma major radius $R_{p}$ ) is given by a certain value of engineering safety factor $q_{\text {eng }}=\pi\left(1+\kappa^{2}\right) a_{p}^{2} B_{t} / \mu_{0} R_{p} I_{p}\left(q_{\text {eng }}=3.5\right.$ in the present study). The elongation $\kappa$ is tentatively chosen as $\kappa=2.0$ at $A=3$ and $\kappa=1.85$ at $A=3.5$ (see Table 1). The maximum toroidal field is determined as $B_{\max } R_{c}=B_{t} R_{p}$, where $R_{c}$ is the inner major radius of a TF coil giving the maximum field. We put a distance between TF coil and plasma, $\Delta_{c}=R_{p}-a_{p}-R_{c}$, to install the vacuum vessel and blanket ( $\Delta_{c}=1.6 \mathrm{~m}$ for simplicity in the present study).

Thus we can make a parameter scan by varying $R_{p}$, and obtain results of Figs. 6 (a) and (c), i.e., $I_{p}, B_{t}$ and $B_{\max }$ for given $A$ and $H_{H}$. The $H_{H}$ value is set constant, $H_{H}=1.2$, for solid lines in Fig. 6, while it is varied for broken lines as $H_{H}=1.1$ at $R_{p}=9 \mathrm{~m}$ and $H_{H}=1.5$ at $R_{p}=7 \mathrm{~m}$ with a linear relation $H_{H}=1.1+0.2\left(9-R_{p}[\mathrm{~m}]\right)$. 
Next we set the operation electron density $n_{e}$ so that the Greenwald-density fraction, $f_{G W}=$ $n_{e} / n_{G W}$ is constant $\left(f_{G W}=0.85\right.$ tentatively in the present study), where $n_{G W}\left[10^{20} \mathrm{~m}^{-3}\right]=I_{p} / \pi a_{p}^{2}$ $\left[\mathrm{MA} / \mathrm{m}^{2}\right]$. It is a future work to find an optimum $f_{G W}$ value for the reactor performance. It is noted that the burning-plasma temperature $T$ is determined mainly by $H_{H} I_{p} A$ value $\left(T \approx 17 \mathrm{keV}\right.$ at $H_{H} I_{p} A \approx$ 70MA; see Table 1). Then the normalized beta, $\beta_{N}=\left(100 a_{p} B_{t} / I_{p}\right)\left(4 \mu_{0} n_{e} T / B_{t}^{2}\right)$, is considered proportional to $a_{p} n_{e} / B_{t} I_{p} \sim f_{G W} / a_{p} B_{t}$ under the constant $H_{H} I_{p} A$ condition. Similarly the fusion power $P_{f}$ is given in proportion to $n_{e}{ }^{2} V_{p} \sim f_{G W}{ }^{2} I_{p}{ }^{2} R_{p} / a_{p}{ }^{2}$, where $V_{p}=2 \pi^{2} \kappa R_{p} a_{p}^{2}$ is plasma volume (a reference value, $P_{f}=3 \mathrm{GW}$ at $A=3, R_{p}=9 \mathrm{~m}$ and $H_{H}=1.1$, is applied in the present study; see Table 1$)$. The neutron wall load is evaluated as $q_{n}=4 P_{f} / 5 S_{w}$, where the wall surface area is simply calculated as $S_{w}=$ $2 \pi^{2}(1+\kappa) R_{p}\left(a_{p}+\Delta_{p}\right)$ with a gap $\Delta_{p}$ between plasma and wall $\left(\Delta_{p}=0.3 \mathrm{~m}\right.$ in the present study). We obtain results of Figs. 6 (b) and (d), i.e., $\beta_{N}, P_{F}$ and $q_{n}$ for given $A$ and $H_{H}$.

\section{REFERENCES}

[1] M. Kikuchi, M. Azumi, Steady State Tokamak Research - Core Physics, Rev. Mod. Phys. 84, 1807(2012)

[2] A. Loarte, G. Saibene, R. Sartori, D. Campbell, et al., Characteristics of type I ELM energy and particle losses in existing devices and their extrapolation to ITER, Plasma Phys. Control, Fusion 45, 1549(2003)

[3] R.J. Goldston, Heuristic drift-based model of the power scrape-off width in low-gas-puff H-mode tokamaks, Nucl. Fusion 52, 013009(2012)

[4] T. Eich, B. Sieglin, A. Scarabosio, W. fundamenski, R.J. Goldston, A. Herrmann, and ASDEX Upgrade Team, Inter-ELM Power Decay Length for JET and ASDEX Upgrade: Measurement and Comparison with Heuristic Drift-Based Model, Phys. Rev. Lett. , 107, 215001(2011)

[5] M. Kikuchi, A. Fasoli, T. Takizuka, P. Diamond, S. Yu Medvedev, et al., Negative Triangularity Tokamak as Fusion Energy System, 1st International E-Conference on Energies March 14, 2014, E002, DOI:10.3390/ECE-1-E002, http://www.sciforum.net/conference/ece-1/paper/2321 
[6] M. Kikuchi, T. Takizuka, Is H-mode relevant for Fusion Reactor, Plenary talk at US-EU TTF 2013 (Santa Rosa, USA, 2013), http://ttf2013.ucsd.edu/TTF_Meeting/Presentations.html

[7] M. Kikuchi, T. Takizuka, M. Furukawa, Negative Triangularity as a Possible Tokamak Scenario, Proc. 12 ${ }^{\text {th }}$ Asia Pacific Physics Conference (July 14-19, 2013, Makuhari), JPS Conf. Proc. 1 (2014)015014

[8] M. Kikuchi, S. Yu Medvedev, T. Takizuka, A. Fasoli, Y. Wu, et al., Perspective of Negative Triangularity Tokamak as Fusion Energy System", 42th EPS Conf. on Plasma Physics and $\begin{array}{lllllll}\text { Controlled Fusion, volume } & \text { ECA } & \text { vol } & 3 & \text { (Lisbon, } & \text { Portugal) } & \text { P4.179 }\end{array}$ (http://ocs.ciemat.es/EPS2015PAP/pdf/P4.179.pdf)

[9] S. Yu Medvedev, M. Kikuchi, L. Villard, T. Takizuka, P. Diamond, et al., The negative triangularity tokamak: stability limits and prospects as a fusion energy system, Nucl. Fusion $\mathbf{5 5}$, $063013(2015)$

[10] S. Yu Medvedev, M. Kikuchi, T. Takizuka, et al., Single Null Divertor in Negative Triangularity Tokamak, $26^{\text {th }}$ IAEA Fusion Energy Conference, 17-22 October 2016, Kyoto, Japan. https://nucleus.iaea.org/sites/fusionportal/Shared\%20Documents/FEC\%202016/FEC2016_ConfM at_Online.pdf , paper EX/P3-47 ; See also M. Kikuchi, S. Medvedev, D. Chen, et al., Single null negative triangularity tokamak reactor concept, $18^{\text {th }}$ International Congress on Plasma Physics (ICPP2016), June 27-July1, Kaohsiung, Taiwan, paper A4A1-1 http://capst.ncku.edu.tw/ISAPS/ICPP2016/Book\%20of\%20Abctracts.pdf

[11] M. Kikuchi, R.W. Conn, F. Najmabadi, Y. Seki, Recent directions in plasma physics and its impact on tokamak magnetic fusion design, Fusion Eng. Design 16, 253 (1991)

[12] G. Federici, C.H. Skinner, et al., Plasma-Material Interactions in current tokamaks and their implications for next step fusion reactors, Nucl. Fusion 41, 1967 (2003)

[13] B.B. Kadomtsev, O.P. Pogutse, Trapped particles in toroidal magnetic systems, Nucl. Fusion 11, $67(1971)$

[14] F. Ryter, C. Angioni, A.G. Peeters, F. Leuterer, W. Suttrop, and ASDEX Upgrade Team, Experimental Study of Trapped-Electron-Mode Properties in Tokamaks: Threshold and Stabilization by Collisions, Phys. Rev. Lett. 95, 085001(2005) 
[15] W.L. Zhong, X.L. Zou, C. Bourdelle, S.D. Song, J.F. Artaud, T. Aniel, X.R. Duan, Convective Velocity Reversal Caused by Turbulence Transition in Tokamak Plasma, Phys. Rev. Lett. 111, 265001(2013)

[16] M.N. Rosenbluth, M.L. Sloan, Finite- $\beta$ Stabilization of the Collisionless Trapped Particle Instability, Phys. Fluids 14, 1725(1971)

[17] J.W. Connor, R.J. Hastie, T.J. Martin, Effect of pressure gradients on the bounce-averaged particle drifts in a tokamak, Nucl. Fusion 23, 1702(1983)

[18] B.B. Kadomtsev, O.P. Pogutse, Reviews of Plasma Physics Vol. 5, (Consultants Bureau, NY, 1970): Also Sov. Phys. JETP 24, 1172(1967)

[19] G. Rewoldt, W.M. Tang, M.S. Chance, Electromagnetic kinetic toroidal eigenmodes for general magnetohydrodynamic equilibria, Phys. Fluids 25, 480(1982)

[20] A. Pochelon, T.P. Goodman, M. Henderson, et al., Energy confinement and MHD activety in shaped TCV plasmas with localized electron cyclotron heating, Nucl. Fusion 39, 1807(1999)

[21] Y. Camenen, A. Pochelon, R. Behn, A. Bottino, A. Bortolon, S. Coda, A. Karpushov, O. Sauter, G. Zhuang and the TCV team, Impact of plasma triangularity and collisionality on electron heat transport in TCV L-mode plasmas, Nucl. Fusion 47, 510 (2007)

[22] M. Fontana, L. Porte, S. Coda, O. Sauter and TCV Team, The effect of triangularity on fluctuations in a tokamak plasma, Nucl. Fusion 58, 024002(2018)

[23] M. Fontana, (2018) Turbulence studies in TCV using the Correlation ECE diagnostic, EPFL PhD thesis No 9016 (https://infoscience.epfl.ch/record/258091/files/EPFL_TH9016.pdf)

[24] O. Sauter, S. Brunner, D. Kim, G. Merlo, R. Behn, Y. Camenen, S. Coda, B.P. Duval, L. Federspiel, T.P. Goodman, A. Karpushov, A. Merle, and TCV Team, On the non-stiffness of edge transport in L-mode tokamak plasmas, Phys. Plasmas 21, 055906(2014)

[25] G. Merlo, S. Brunner, O. Sauter, Y. Camenen, T. Gorler, F. Jenko, A. Marinoni, D. Told, L. Villard, Investigating profile stiffness and critical gradients in shaped TCV discharges using local gyrokinetic simulations of turbulent transport, Plasma Phys. Control. Fusion 57, 054010(2015) 
[26] F. Riva, E. Lanti, S. Jolliet, P. Ricci, Plasma shaping effects on tokamak scrape-off layer turbulence, Plasma Phys. Control. Fusion 99, 035001(2017)

[27] M. Austin, A. Marinoni, M.L. Walker, et al., High confinement in negative triangularity discharges in DIII-D, 59 ${ }^{\text {th }}$ APS-DPP Bulletin Bo4 2 (2017)

[28] A. Marinoni, H-mode-like confinement with L-mode edge in negative triangularity plasmas on DIII-D, Abstract MF-I54 in $1^{\text {st }}$ Asia-Pacific Conference on Plasma Physics, 18-23 September, 2017, Chengdu, China. http://aappsdpp.org/DPPP2017rogramlatest/pdf/MF-I54.pdf

[29] M. Austin, A. Marioni, et al., High Confinement in Negative Triangularity Discharges in DIIID, 27 ${ }^{\text {th }}$ IAEA Fusion Energy Conference, 22-27 October 2018, Ahmedabad, India, https://nucleus.iaea.org/sites/fusionportal/Shared\%20Documents/FEC\%202018/FEC2018 ConfM at_Online.pdf, paper EX/P6-6

[30] A. Marinoni, et al., APS-DPP 2018 Invited talk, to be submitted to Physics of Plasmas.

[31] H. Nakamura, et al., H-mode experiments with outer and lower divertors in JT-60, Nuclear Fusion 30(1990)235

[32] T. Ohkawa, V.S. Chan, M.S. Chu, R.R. Dominguez, R.L. Miller, New Current Drive and Confinement Techniques for Improved Tokamak Operation, In Plasma Physics and Controlled Nuclear Fusion Research (Proc. $12^{\text {th }}$ Int. Conf. Nice, 1988), IAEA, Vienna (1989), Vol. 1, p. 681

[33] M.D. Kruskal, C.R. Oberman, On the Stability of Plasma in Static Equilibrium, Phys. Fluids 1, 275(1958)

[34] F. Porcelli, M.N. Rosenbluth, Modified Mercier criterion, Plasma Phys. Control. Fusion 40, 481(1998)

[35] A. Merle, O. Sauter, S. Yu Medvedev, Pedestal properties of H-modes with negative triangularity using the EPED-CH model, Plasma Phys. Control. Fusion 59, 104001(2017)

[36] A. Loarte and G. Federici, private communication.

[37] Y. Takahashi, T. Isono, K. Hamada, et al., Technology development and mass production of $\mathrm{Nb}_{3} \mathrm{Sn}$ conductors for ITER toroidal field coils in Japan, Nucl. Fusion 51, 113015 (2011)

[38] L. Bottura, B. Bordini, $J_{c}(B, T, \varepsilon)$ Parametrization for the ITER $N b_{3} S n$ Production, IEEE Trans. Appl. Supercond., 19(2009)1521 
[39] J-M Rey, A. Allais, J-L Duchateay, et al., Critical current measurement in HTS Bi2212 ribbons and round wires, IEEE Trans. Appl. Superconduct., 19(2009)3088

[40] T. Ando, H. Nakajima, H. Hiue, and Y. Wadayama., The Effect of Ti conduit on the critical current in $\left(\mathrm{N}_{\mathrm{b}} \mathrm{T}_{\mathrm{i}}\right)_{3} \mathrm{~S}_{\mathrm{n}} \quad$ cable-in-donduit conductors, IEEE Transactions on Applied Superconductivity Vol. 3, pp. 488-491 (1993)

[41] N. Cheverev, et al., ITER TF conductor insert manufacturing, IEEE Trans. Appl. Superconductivity, vol. 12, pp. 548-553 (2002)

[42] N. Martovetsky, et al., Test of the ITER TF insert and Central Solenoid Model Coil, IEEE Trans. Appl. Superconductivity, vol. 13, pp. 1441-1446 (2003)

[43] S. Nishio, K. Tobita, S. Konishi, T. Ando, et al., Tight aspect ratio tokamak power reactor with superconducting TF coils, IAEA-CN-FT/P1-21, in $19^{\text {th }}$ IAEA Fusion Energy Conference (Lyon, France, Oct. 2002)

[44] T. Ando, S. Nishio, Design of high $T_{c}$ superconducting TF coil for the tight aspect ratio tokamak power plant, IEEE Trans. Appl. Superconduct., vol 14, 1481(2004)

[45] T. Ando, S. Nishio, Design of the TF coil for a tokamak fusion power reactor with YBCO tape superconductors, Fusion Technology (2006)

[46] J. Jiang et al. (MagLab), August 2017 unpublished (National magnetic field laboratory homepage:https://nationalmaglab.org/magnet-development/applied-superconductivitycenter/plots)

[47] O. Sauter, Geometric formulas for system codes including the effect of negative triangularity, Fusion Engineering and Design 112, 633(2016)

[48] D. Chen, J. Jiang, Y. Hou, W. Duan, M. Ni, C. Xing, Preliminary Cost Assessment and Compare of China Fusion Engineering Test Reactor, J. Fusion Energy (2015) 34:127-132. DOI 10.1007/s10894-014-9770-x

[49] T. Takizuka, S. Tokunaga, K. Hoshino, K. Shimizu, N. Asakura, Combination of helical ferritic-steel inserts and flux-tube expansion divertor for the heat control in tokamak DEMO reactor, J. Nucl. Materials 463, 1229(2015) 
[50] A. Herrmann, Overview on stationary and transient divertor heat loads, Plasma Phys. Control. Fusion 44, 883(2002)

[51] H. Zohm, C. Angioni, E. Fable, G. Federici, et al., On the physics guidelines for a tokamak DEMO, Nucl. Fusion 53, 073019(2013)

[52] L. Villard, et al., Global turbulence features across marginality and nonlocal pedestal-core interactions, Plasma Phys. Contr. Fusion, to be published.

[53] E.J. Doyle, W.A. Houlberg, et al., Progress in the ITER Physics Basis Chapter 2, Plasma confinement and transport, Nucl. Fusion 47, S18(2007)

[54] S. Sengoku, A. Funahashi, et al., Regime of Improved Confinement in Neutral-Beam-Heated Limiter Discharges of a Tokamak, Phys. Rev. Lett. 59, 450 (1987)

[55] S. Tsuji, K. Ushigusa, et al., Observation of the Limiter H-mode in the JT-60 Tokamak with Lower-Hybrid Current Drive, Phys. Rev. Lett. 64, 1023 (1990)

[56] K. Toi, K. Kawahata, et al., Role of Edge Magnetic Shear on the Limiter H-mode Transition of the JIPP T-IIU Tokamak, Phys. Rev. Lett. 64, 1895 (1990)

[57] J. Moret, M. Anton, et al., Ohmic H-mode and confinement in TCV, Plasma Phys. Contr. Fusion 37, A215(1995)

[58] M. Kikuchi, M. Azumi, Frontier in Fusion Research II - Introduction to Modern Tokamak Physics, Springer (2015)

[59] The National Academy of Sciences, Final Report of the Committee on a Strategic Plan for U.S. Burning Plasma Research, December 13, 2018. https://www.nap.edu/catalog/25331/final-reportof-the-committee-on-a-strategic-plan-for-us-burning-plasma-research

[60] K. Hoshino, N. Asakura, K. Shimizu, et al., Divertor Study on DEMO Reactor, Plasma and Fusion Research, 9, 3403070 (2014)

[61] ITER Physics Basis Editors, ITER Physics Expert Group Chairs and Co-Chairs, ITER JCT and Physics Integration Unit, ITER Physics Basis, Chapter 1: Overview and summary, Appendix A, Nucl. Fusion 39, 2137 (1999). 
[62] ITER Physics Expert Groups on Confinement \& Transport and Confinement Modelling \& Database and ITER Physics Basis Editors, ITER Physics Basis, Chapter 2: Plasma confinement and transport, Nucl. Fusion 39, 2175 (1999). 
Table 1 Major coil parameters of three TF coil designs in Figure 5

\begin{tabular}{|l|l|l|l|}
\hline & $\mathrm{A}=3, \mathrm{R}_{\mathrm{p}}=9 \mathrm{~m}$ & $\mathrm{~A}=3, \mathrm{R}_{\mathrm{p}}=7 \mathrm{~m}$ & $\mathrm{~A}=3.5, \mathrm{R}_{\mathrm{p}}=7 \mathrm{~m}$ \\
\hline Coil shape & Racetrack & Racetrack & Racetrack \\
\hline Coil size & $15 \mathrm{~m} \times 20 \mathrm{~m}$ & $12 \mathrm{~m} \times 16 \mathrm{~m}$ & $11 \mathrm{~m} \times 14 \mathrm{~m}$ \\
\hline Number of coils & 18 & 18 & 18 \\
\hline Magnetic Energy & $170 \mathrm{GJ}$ & $86.5 \mathrm{GJ}$ & $104 \mathrm{GJ}$ \\
\hline Maximum field & $13.6 \mathrm{~T}$ & $13.5 \mathrm{~T}$ & $15.5 \mathrm{~T}$ \\
\hline Ampere Turn & $263 \mathrm{MAT}$ & $207 \mathrm{MAT}$ & $263 \mathrm{MAT}$ \\
\hline Discharge constant & $12 \mathrm{~s}$ & $10 \mathrm{~s}$ & $12 \mathrm{~s}$ \\
\hline Quench detection & $2 \mathrm{~s}$ & $2 \mathrm{~s}$ & $2 \mathrm{~s}$ \\
\hline Temperature rise & $150 \mathrm{~K}$ & $150 \mathrm{~K}$ & $150 \mathrm{~K}$ \\
\hline Number of Turn/coil & 116 & 116 & 146 \\
\hline Conductor current & $126 \mathrm{kA}$ & $100 \mathrm{kA}$ & $100 \mathrm{kA}$ \\
\hline Winding structure & ITER-like & ITER-like & ITER-like \\
\hline Disk per coils & 6 & 6 & 7 \\
\hline Terminal voltage & $12.5 \mathrm{kV}$ & $10 \mathrm{kV}$ & $10 \mathrm{kV}$ \\
\hline
\end{tabular}


Table $2 \mathrm{TF}$ conductor designs of three reactor configurations in Figure 5 ( $A=3, R_{p}=9 \mathrm{~m} ; A=3$, $\left.R_{p}=7 m ; A=3.5 R_{p}=7 \mathrm{~m}\right)$

\begin{tabular}{|c|c|c|c|c|c|c|}
\hline & \multicolumn{2}{|c|}{$\mathrm{A}=3, \mathrm{R}_{\mathrm{p}}=9 \mathrm{~m}$} & \multicolumn{2}{|c|}{$\mathrm{A}=3, \mathrm{R}_{\mathrm{p}}=7 \mathrm{~m}$} & \multicolumn{2}{|c|}{$\mathrm{A}=3.5, \mathrm{R}_{\mathrm{p}}=7 \mathrm{~m}$} \\
\hline Conductor type & Nb3Sn CIC(SS) & Nb3Sn CIC (Ti) & Nb3Sn CIC(SS) & Nb3Sn CIC (Ti) & $\mathrm{Nb} 3 \mathrm{Sn} \mathrm{CIC}$ & $\mathrm{Bi} 2212$ \\
\hline Nominal field & $13.6 \mathrm{~T}$ & $13.6 \mathrm{~T}$ & $13.5 \mathrm{~T}$ & $13.5 \mathrm{~T}$ & $15.5 \mathrm{~T}$ & $15.5 \mathrm{~T}$ \\
\hline Total operating strain & $-0.6 \%$ & $-0.05 \%$ & $-0.6 \%$ & $-0.05 \%$ & $-0.05 \%$ & $0.2 \%$ \\
\hline Current sharing Temp. & $6.1 \mathrm{~K}$ & $6.2 \mathrm{~K}$ & $6.1 \mathrm{~K}$ & $6.1 \mathrm{~K}$ & $6.2 \mathrm{~K}$ & $21.2 \mathrm{~K}$ \\
\hline Operating curr./Crit. curr. & 0.64 & 0.56 & 0.61 & 0.56 & 0.72 & 0.89 \\
\hline Central coolant & Supercritical He & Supercritical He & Supercritical He & Supercritical He & Supercritical He & Gas He \\
\hline Conductor outer diameter & $66.0 \mathrm{~mm}$ & $61.8 \mathrm{~mm}$ & $60.0 \mathrm{~mm}$ & $54.8 \mathrm{~mm}$ & $58.0 \mathrm{~mm}$ & $58.6 \mathrm{~mm}$ \\
\hline Jacket material & SS & $\mathrm{Ti}$ & SS & $\mathrm{Ti}$ & $\mathrm{Ti}$ & No jacket \\
\hline $\mathrm{SC}$ and $\mathrm{Cu}$ strand diameter & $0.84 \mathrm{~mm}$ & $0.87 \mathrm{~mm}$ & $0.84 \mathrm{~mm}$ & $0.76 \mathrm{~mm}$ & $0.81 \mathrm{~mm}$ & $0.82 \mathrm{~mm}$ \\
\hline $\mathrm{Cu} / \mathrm{Non} \mathrm{Cu}, \mathrm{Ag} /$ non $\mathrm{Ag}$ & 1.0 & 2.0 & 1.0 & 2.0 & 1.6 & 0.82 \\
\hline Cabling pattern & $3 \times 3 \times 4 \times 4 \times 4 \times 6$ & $3 \times 3 \times 3 \times 4 \times 4 \times 6$ & $3 \times 3 \times 3 \times 4 \times 4 \times 6$ & $3 \times 3 \times 3 \times 4 \times 4 \times 6$ & $3 \times 3 \times 3 \times 4 \times 4 \times 6$ & $3 \times 3 \times 3 \times 4 \times 4 \times 6$ \\
\hline
\end{tabular}


Table 3 Steady-state operation parameters from the system code corresponding to three cases in Figure 5. Temperature and density profiles are assumed as $\mathrm{T}(\mathrm{r})=\mathrm{T}_{0}\left(1-(\mathrm{r} / \mathrm{a})^{2}\right) 1.3, \mathrm{n}(\mathrm{r})=\mathrm{n}_{0}[0.9(1-$ $\left.\left.\left.(\mathrm{r} / \mathrm{a})^{2}\right)\right]^{0.5}+0.1\right]$.

\begin{tabular}{|c|c|c|c|c|c|}
\hline No & Input Parameter & Symbol & $A=3, R_{p}=9 m$ & $\mathrm{~A}=3, \mathrm{R}_{\mathrm{p}}=7 \mathrm{~m}$ & $\mathrm{~A}=3.5, \mathrm{R}_{\mathrm{p}}=7 \mathrm{~m}$ \\
\hline 1 & Plasma current (MA) & $\mathrm{I}_{\mathrm{p}}$ & 21 & 15.4 & 13.2 \\
\hline 2 & Toroidal field $(\mathrm{T})$ & $\mathrm{B}_{\mathrm{t}}$ & 5.86 & 5.94 & 7.53 \\
\hline 3 & Major radius (m) & $\mathrm{R}_{\mathrm{p}}$ & 9.0 & 7.0 & 7.0 \\
\hline 4 & Aspect Ratio & A & 3.0 & 3.0 & 3.5 \\
\hline 5 & Elongation at separatrix & $\kappa_{\mathrm{x}}$ & 2.0 & 2.0 & 1.85 \\
\hline 6 & Upper and lower triangularity at sep. & $\delta_{u} / \delta_{1}$ & $-0.4 /-0.9$ & $-0.4 /-0.9$ & $-0.4 /-0.9$ \\
\hline 7 & Normalized beta & $\beta_{\mathrm{N}}$ & 2.1 & 2.7 & 2.5 \\
\hline 8 & Greenwald fraction & $\mathrm{f}_{\mathrm{GW}}$ & 0.85 & 0.85 & 0.85 \\
\hline 9 & Confinement enhancement over y2 scaling & $\mathrm{H}_{\mathrm{H}}$ & 1.12 & 1.5 & 1.5 \\
\hline 10 & Helium fraction & $\mathrm{f}_{\mathrm{He}}$ & 0.05 & 0.05 & 0.05 \\
\hline 11 & Impurity fraction & $\mathrm{f}_{\mathrm{imp}}$ & 0.002 & 0.0019 & 0.002 \\
\hline 12 & Current drive efficiency $\left(10^{20} \mathrm{~A} / \mathrm{W} / \mathrm{m}^{2}\right)$ & $\eta_{\mathrm{CD}}$ & 0.5 & 0.5 & 0.5 \\
\hline No & Output parameters & Symbol & $\mathrm{A}=3, \mathrm{R}_{\mathrm{p}}=9 \mathrm{~m}$ & $\mathrm{~A}=3, \mathrm{R}_{\mathrm{p}}=7 \mathrm{~m}$ & $\mathrm{~A}=3.5, \mathrm{R}_{\mathrm{p}}=7 \mathrm{~m}$ \\
\hline 1 & Minor radius (m) & $\mathrm{a}_{\mathrm{p}}$ & 3.0 & 2.333 & 2.0 \\
\hline 2 & Cylindrical safety factor & $\mathrm{q}_{\mathrm{cy}}$ & 3.49 & 3.75 & 3.60 \\
\hline 3 & Bootstrap current fraction & $\mathrm{f}_{\mathrm{bs}}$ & 0.30 & 0.41 & 0.39 \\
\hline 4 & Effective Charge & $Z_{\text {eff }}$ & 1.73 & 1.67 & 1.71 \\
\hline 5 & Line average density $\left(\alpha_{n}=0.5\right)\left(10^{20} \mathrm{~m}^{-3}\right)$ & $\bar{n}_{e}$ & 0.63 & 0.77 & 0.89 \\
\hline 6 & Volume av. temperature $\left(\alpha_{\mathrm{T}}=1.3\right)(\mathrm{keV})$ & $<\mathrm{T}_{\mathrm{e}}>,<\mathrm{T}_{\mathrm{i}}>$ & 16.9 & 17.1 & 17.2 \\
\hline 7 & Plasma volume $\left(\mathrm{m}^{3}\right)$ & $\mathrm{V}_{\mathrm{p}}$ & 3235 & 1522 & 1025 \\
\hline 8 & Plasma surface $\left(\mathrm{m}^{2}\right)$ & $\mathrm{S}_{\mathrm{p}}$ & 1789 & 1082 & 870 \\
\hline 9 & Fusion power (MW) & $\mathrm{P}_{\mathrm{F}}$ & 2964 & 2053 & 1862 \\
\hline 10 & Neutron flux $\left(\mathrm{MW} / \mathrm{m}^{2}\right)$ & $\Gamma_{\mathrm{n}}$ & 1.33 & 1.52 & 1.71 \\
\hline 11 & Total heating power (MW) & $\mathrm{P}_{\text {tot }}$ & 761 & 507 & 473 \\
\hline 12 & Current drive power (MW) & $\mathrm{P}_{\mathrm{CD}}$ & 168 & 96.6 & 101 \\
\hline 13 & Transport loss power (MW) & $\mathrm{P}_{\text {transp }}$ & 594 & 384 & 338 \\
\hline 14 & Radiation loss power (MW) & $\mathrm{P}_{\mathrm{rad}}$ & 167 & 124 & 135 \\
\hline 15 & Energy gain & Q & 17.7 & 21.3 & 18.5 \\
\hline 16 & Energy confinement time (s) & $\tau_{\mathrm{E}}$ & 2.80 & 2.51 & 2.24 \\
\hline 17 & Poloidal beta & $\beta_{p}$ & 1.08 & 1.50 & 1.57 \\
\hline 18 & Toroidal beta (\%) & $\beta_{t}$ & 2.51 & 3.0 & 2.19 \\
\hline
\end{tabular}




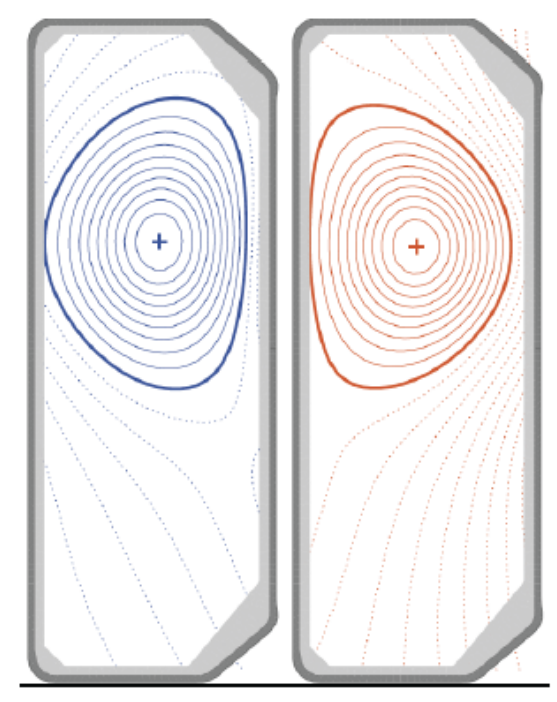

$\operatorname{TCV}\left(\mathrm{a}_{\mathrm{p}}=25 \mathrm{~cm}, \mathrm{~B}_{\mathrm{t}}=1.44 \mathrm{~T}\right)$
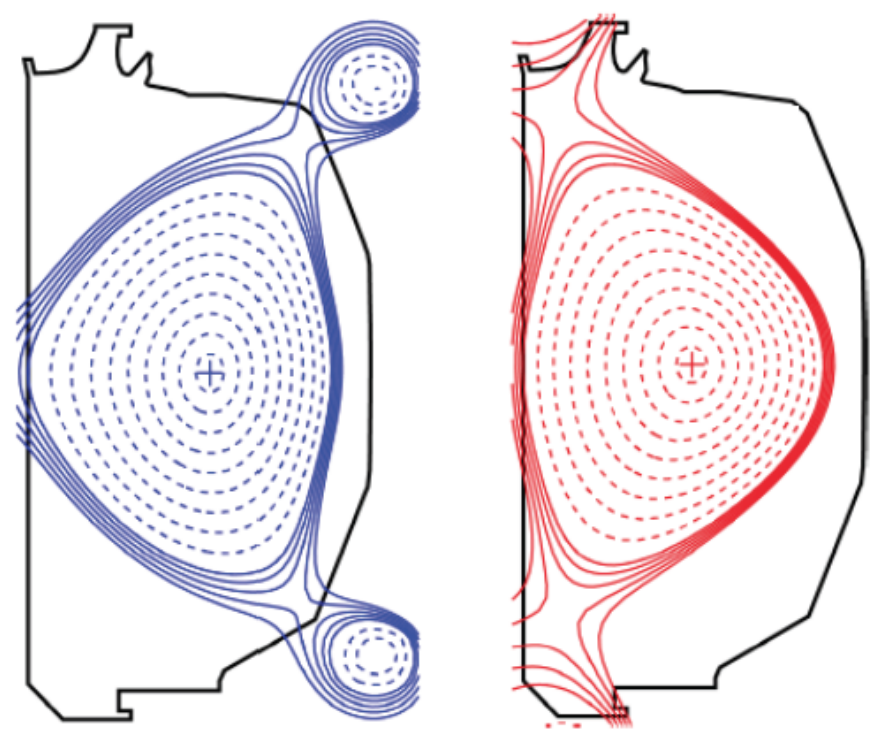

DIII-D $\left(a_{p}=59 c m, B_{t}=2 T\right)$

Figure 1 D-shaped and NTT experimental configurations in TCV (PT:S=9.9m ${ }^{2}$, NT: $\left.10.3 m^{2}\right)$ and DIIID (PT: $S=42 m^{2}$, NT: $\left.S=45 m^{2}\right)$. Here $S$ is plasma surface area. 
a) Optimized pressure profile

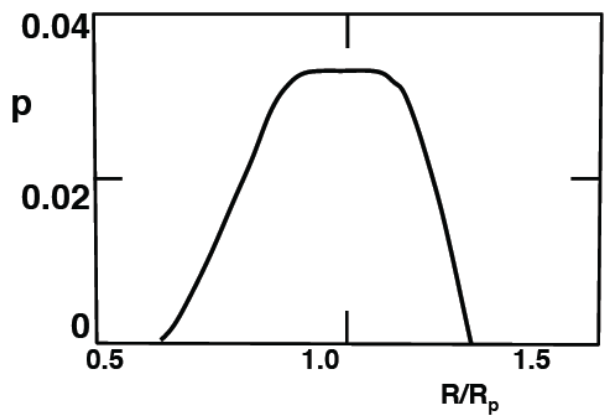

b) Optimized pressure gradient profile in KINX

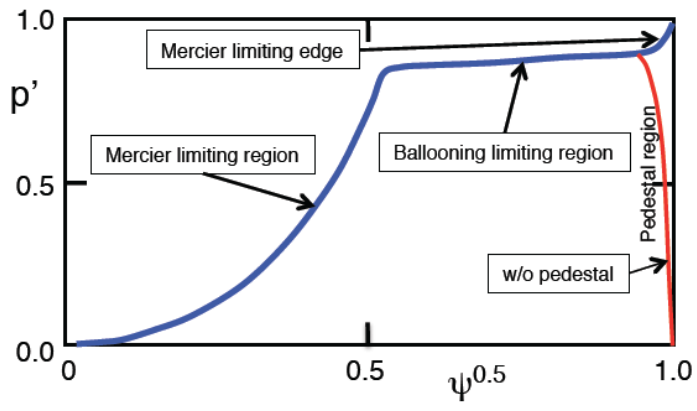

Figure 2 a) Optimized pressure profile in NTT. Near-flat profile near the plasma center. b) Mercier limiting region and ballooning limiting region. Comparison of Mercier limiting edge and zero gradient edge. 


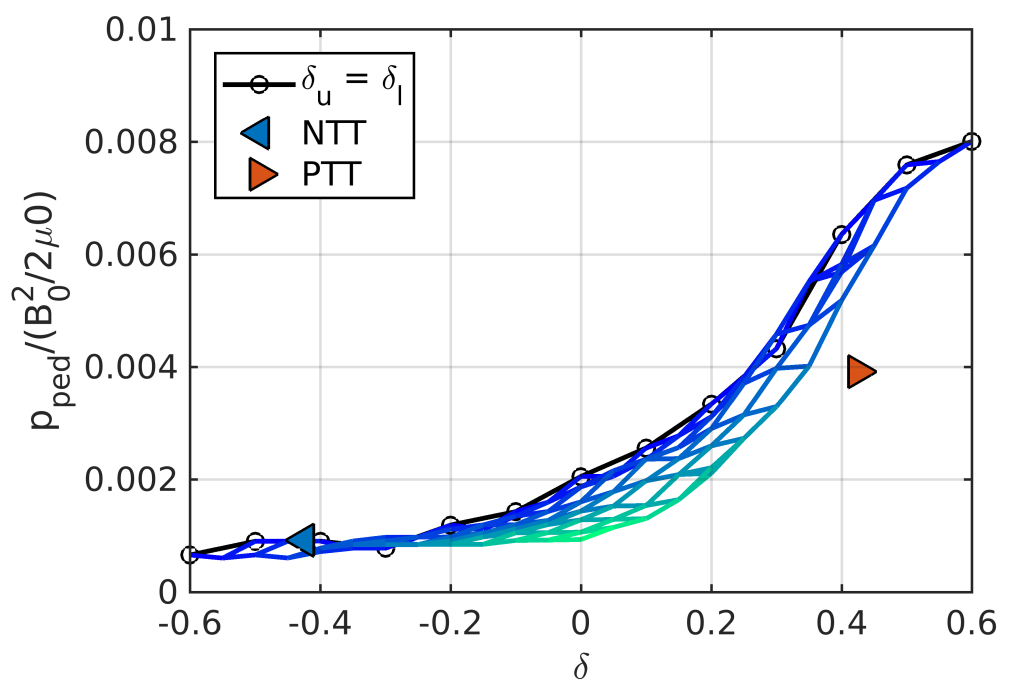

Figure 3 Normalized pedestal pressure limit determined by EPED-CH code as a function of average triangularity $\delta$. Parameters used are $R_{p}=9 m, a_{p}=3 m, B_{t}=5 T, I_{p}=15 M A, \kappa_{x}=1.8, \delta_{l}=-0.85, \delta_{u}=0$ for the NTT and $\delta_{l}=+0.85, \delta_{u}=0$ for the standard D-shaped plasma [30]. The color indicates deviation from up-down symmetry going from blue to green for more asymmetry(color online). 


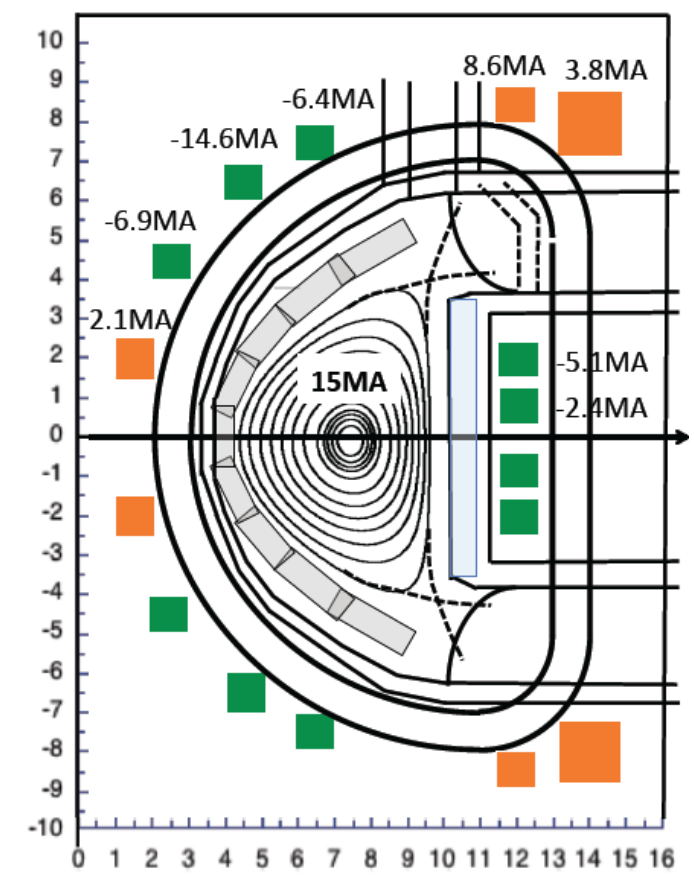

Figure 4 Inverted D-shaped Toroidal Field Coil for original $\kappa_{\mathrm{x}}=1.5 \mathrm{DN}-\mathrm{NTT}$ configuration $\left(\mathrm{R}_{\mathrm{p}}=7 \mathrm{~m}\right.$, $\mathrm{a}_{\mathrm{p}}=2.7 \mathrm{~m}, \delta=-0.9, \mathrm{I}_{\mathrm{p}}=15 \mathrm{MA}, \mathrm{B}_{\mathrm{t}}=6.2 \mathrm{~T}$. 


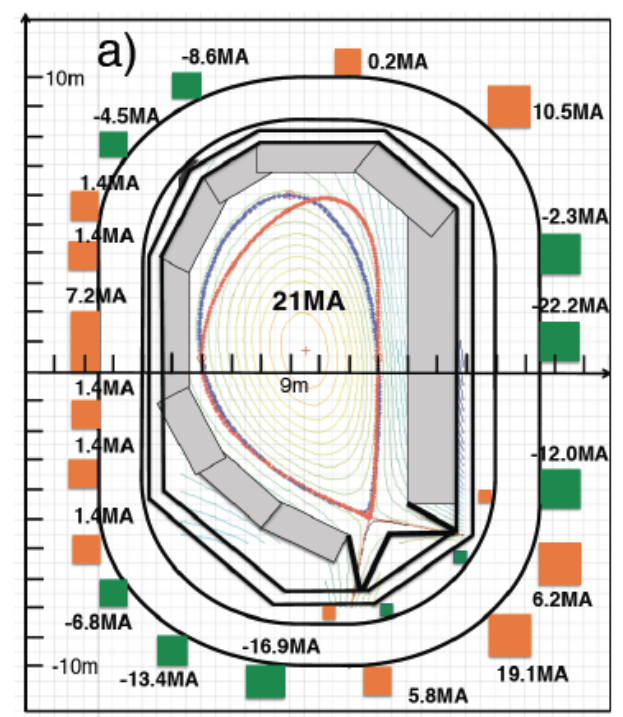

b)

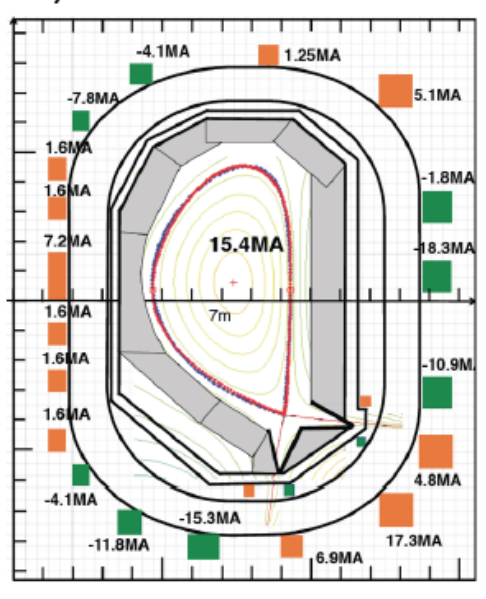

c)

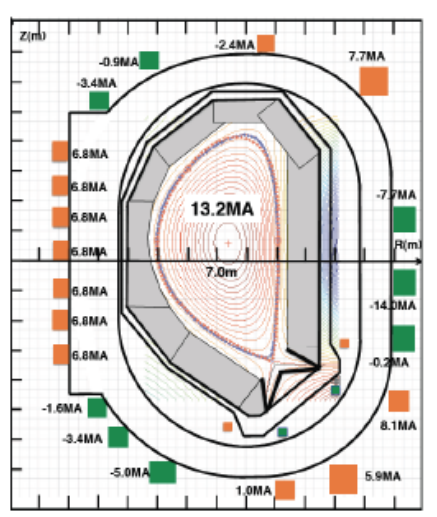

Figure 5 Configuration designs of NTT reactors. Here, green-colored PF coils are for pushing and orange-colored PF coils are for attracting (color online).

a) $A=3$ conservative design $\left(I_{p}=21 M A, B_{t}=5.86 T, B_{\max }=13.6 T, R_{p}=9 m, \delta_{u}=-0.4, \delta_{l}=-0.9, \kappa_{x}=1.8\right.$, $\left.l_{i}=0.84, \beta_{N}=2.1\right)[10]$,

b) $A=3$ high confinement $\left(I_{p}=15.4 M A, B_{t}=5.94 T, B_{\max }=13.5 T, R_{p}=7 m, \delta_{u}=-0.4, \delta_{l}=-0.9, \kappa_{x}=2.0, H_{H}\right.$ $=1.50, l_{i}=0.85, \beta_{N}=2.7, \beta_{N}^{N W}=2.89$ ),

c) $A=3.5$ high field and high confinement $\left(R_{p}=7 m, \delta_{u}=-0.4, \delta_{l}=-0.9, \kappa_{x}=1.85, H_{H}=1.5, B_{t}=7.53 T\right.$, $B_{\max }=15.5 T, l_{i}=0.91, \beta_{N}=2.5, \beta_{N}{ }^{N W}=2.87$ ). 
a) $A=3.0, H_{H} I_{p} A=69.3 \mathrm{MA}$

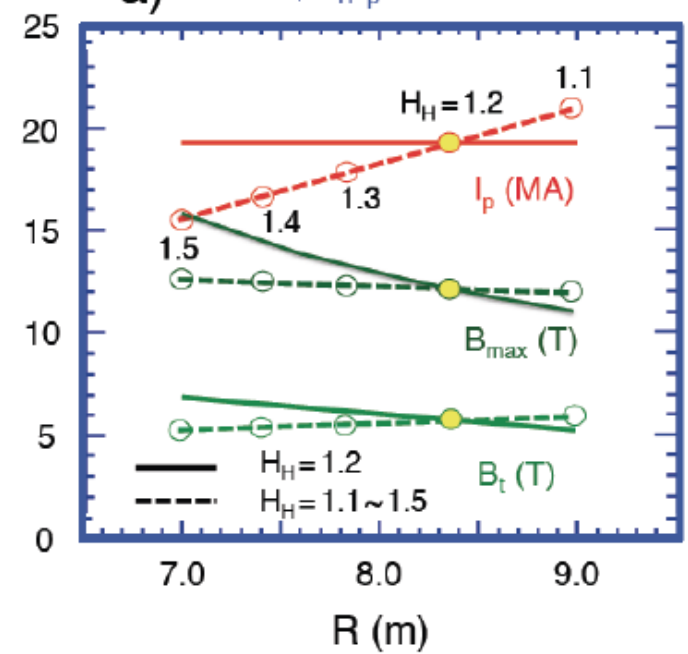

b) $A=3.0, H_{H} I_{p} A=69.3 \mathrm{MA}$

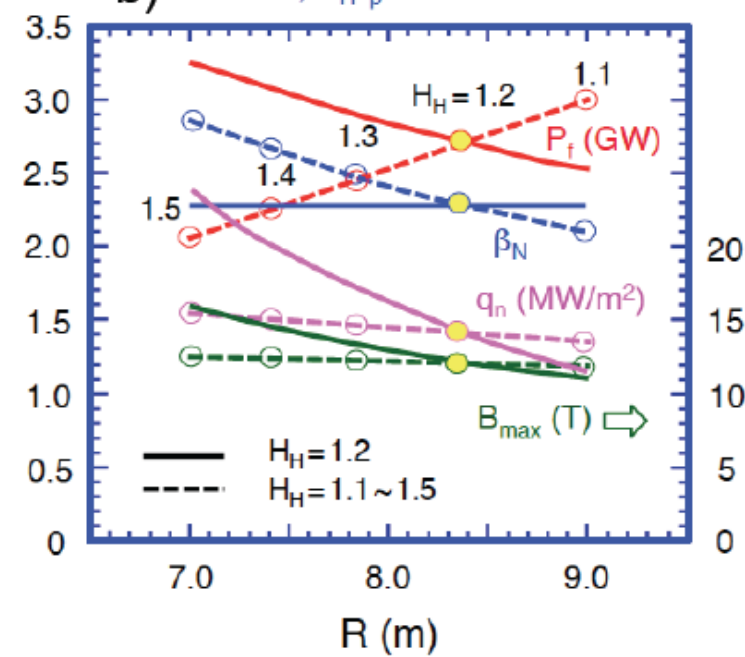

C) $\mathrm{A}=3.5, \mathrm{H}_{\mathrm{H}} \mathrm{I}_{\mathrm{p}} \mathrm{A}=69.3 \mathrm{MA}$

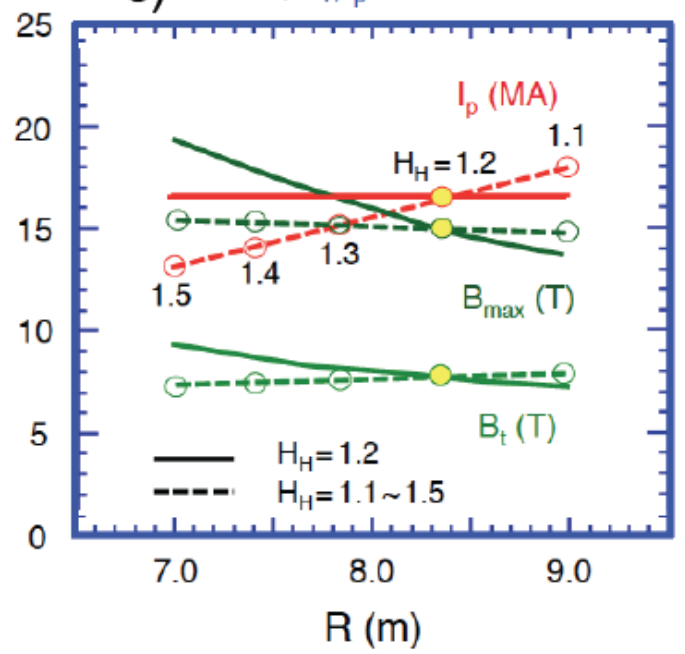

d) $A=3.5, H_{H} I_{p} A=69.3 \mathrm{MA}$

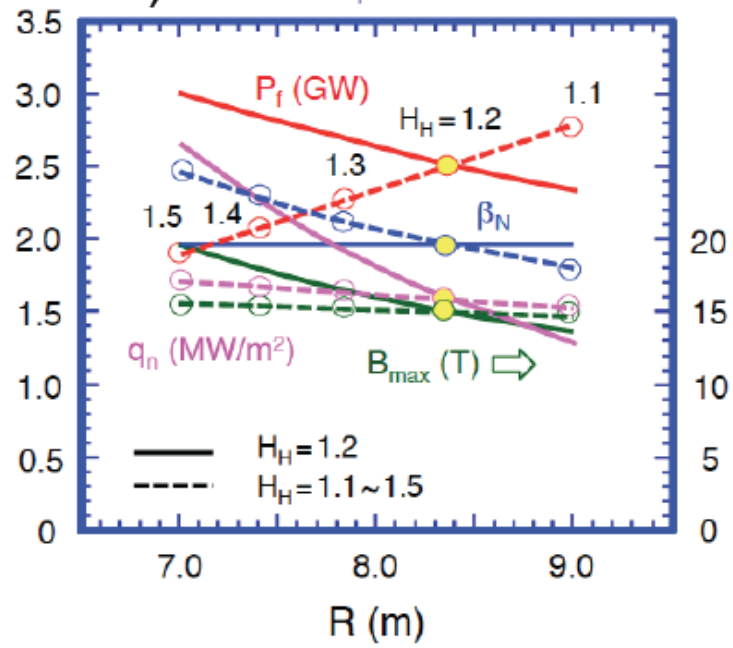

Figure 6 Parameter surveys of NTT reactor designs. a) $R_{p}$ scan of $I_{p}, B_{\max }, B_{t}$ for fixed $H_{H}=1.2$ (solid line) and $H_{H}=1.1-1.5$ (broken line) $(A=3)$, b) $R_{p}$ scan of $P_{f}, \beta_{N}, q_{n}$ for fixed $H_{H}=1.2$ (solid line) and $H_{H}=1.1-1.5$ (broken line) (A=3), c): $R_{p}$ scan of $I_{p}, B_{\max }, B_{t}$ for fixed $H_{H}=1.2$ (solid) and $H_{H}=1.1-1.5$ (broken) $(A=3.5)$, d) $R_{p}$ scan of $P_{f}, \beta_{N}, q_{n}$ for fixed $H_{H}=1.2$ (solid line) and $H_{H}=1.1-1.5$ (broken line) $(A=3.5)$. 


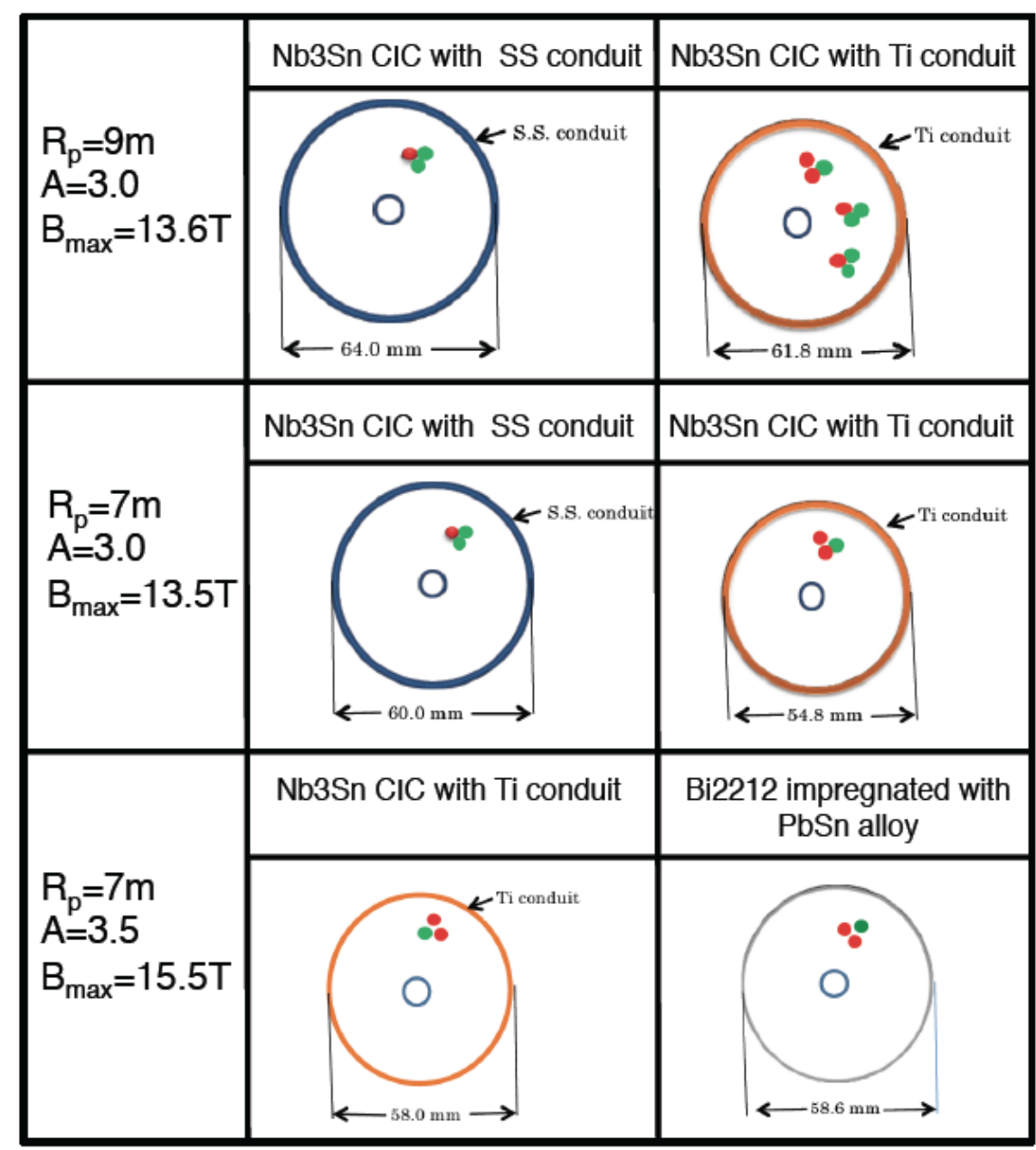

Figure 7 Conductor designs of three reactor configurations. The blue circle is $S S$ conduit and the orange circle is $T_{i}$ conduit. Green closed circle is superconducting strand and the red closed circle is $\mathrm{Cu}$ strand for low temperature SC and $A g$ strand for high $\mathrm{T}_{\mathrm{c}} \mathrm{SC}$ (Bi2212) (color online). 
15MA equilibrium a) without FTE,

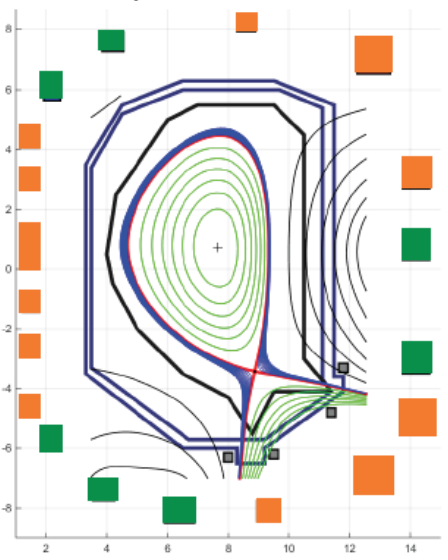

b) with FTE,

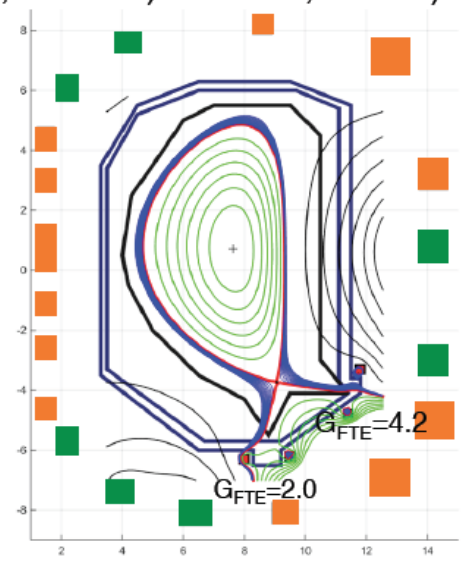

c)Expanded magnetic structure with FTE

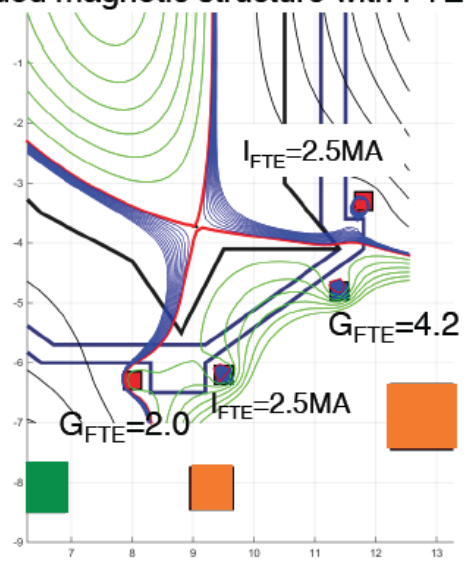

Figure 8 Divertor geometry of $I p=15 M A, R p=7 m$ equilibrium without FTE and with FTE coil current $\left(I_{F T E}=2.5 M A\right)$. Flux tube expansion by 2.0 and 4.2 are obtained for inner and outer divertor legs, respectively. 


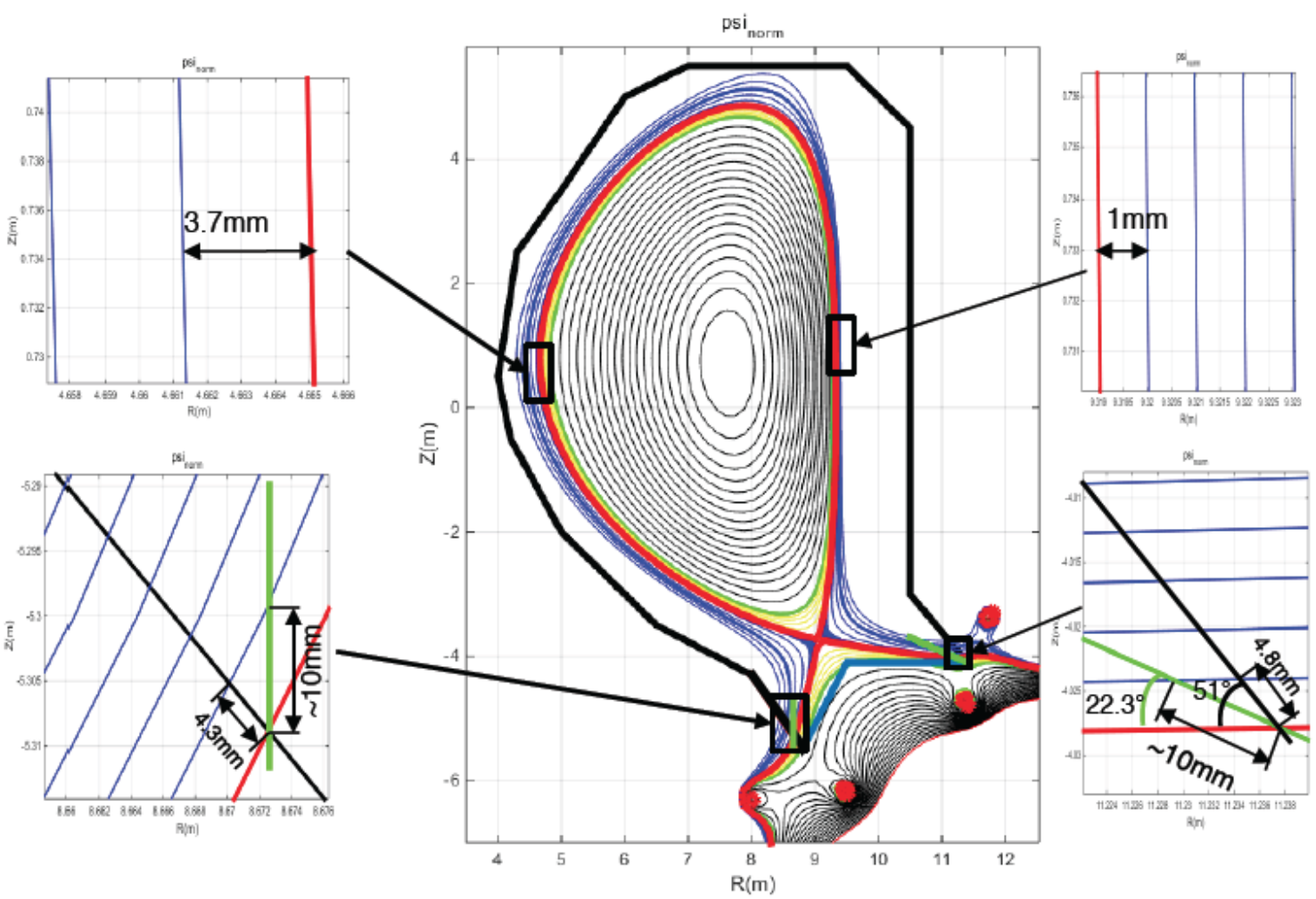

Figure 9 Divertor geometries for NTT $\left(I_{p}=15 M A, R_{p}=7 m, I_{F T E}=2.5 M A\right)$. For a $1 \mathrm{~mm} \mathrm{SOL}$ width at outboard mid-plane, inboard mid-plane thickness is $3.7 \mathrm{~mm}$. Use of FTE coils expanded the flux tube and 4-5 $\mathrm{mm}$ along the divertor plate for the original semi-open divertor target geometry (black lines). With more inclined divertor targets, $1 \mathrm{~mm}$ SOL at outboard mid-plane will be projected to $10 \mathrm{~mm}$ along the divertor plates (green lines) (color online). 


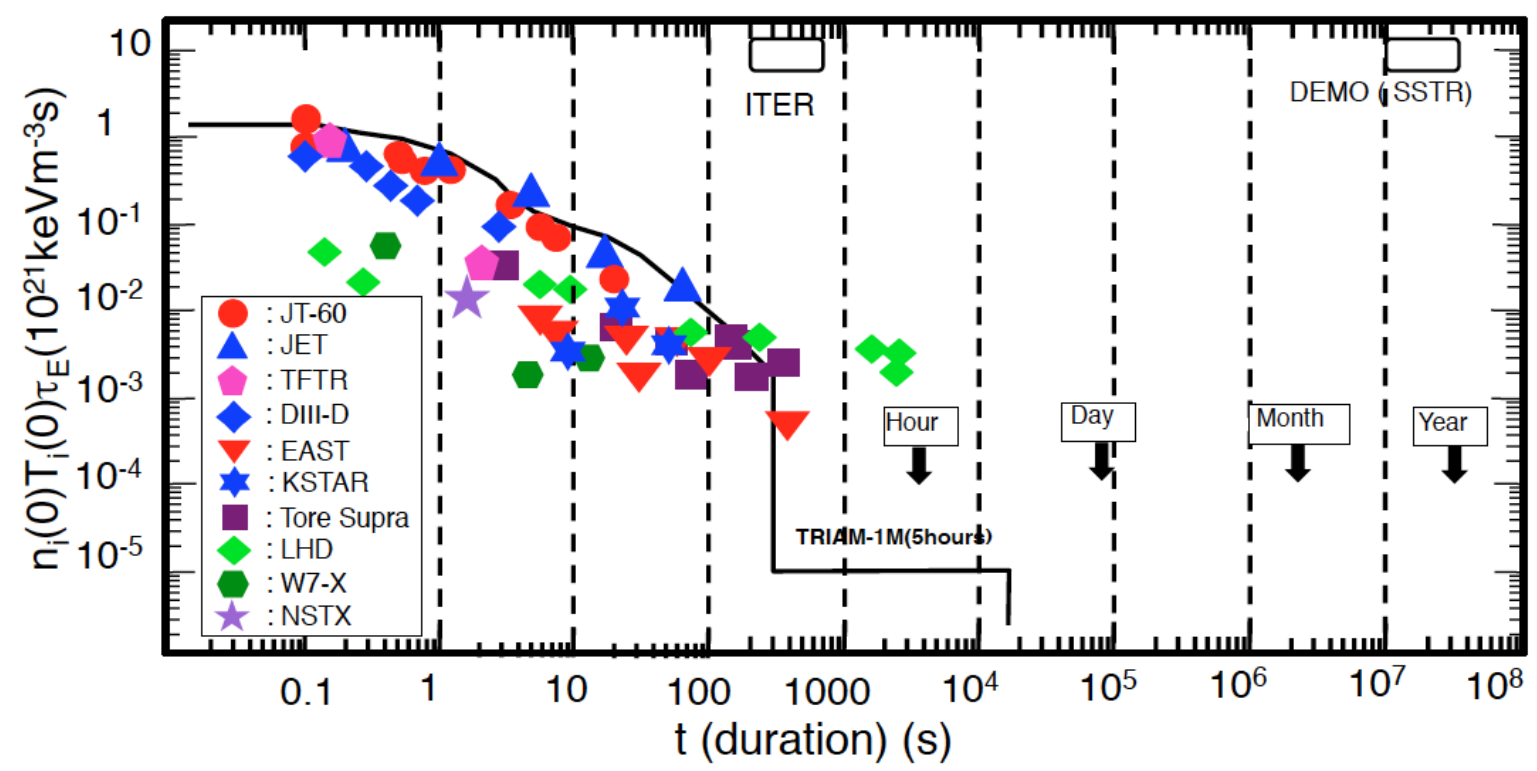

Figure 10 Achieved fusion triple product $\left(n_{i}(0) T_{i}(0) \tau_{E}\right)$ as a function of sustained duration [53]. Data points are added for the US NAS report contributed from B. Wan, H. Park, T. Pedersen, P. Snyder, S. Kaye [54]. 